\title{
An Absolutely New Attempt to Vague Soft Bitopological Spaces Basis at Newly Defined Operations regarding Vague Soft Points
}

\author{
Arif Mehmood, ${ }^{1}$ Farkhanda Afzal $\left(\mathbb{D},{ }^{2}\right.$ Saleem Abdullah $\mathbb{D},{ }^{3}$ Muhammad Imran Khan, ${ }^{4}$ \\ and Saeed Gul $\mathbb{D}^{5}$ \\ ${ }^{1}$ Department of Mathematics, University of Science and Technology Bannu, Bannu, Pakistan \\ ${ }^{2}$ Department of Humanities and Basic Sciences, MCS, National University of Sciences and Technology, Islamabad, Pakistan \\ ${ }^{3}$ Department of Mathematics, Abdul Wali Khan University Mardan, Mardan 23200, Pakistan \\ ${ }^{4}$ Department of Pure and Applied Mathematics (Statistics), University of Haripur, Haripur, Pakistan \\ ${ }^{5}$ Faculty of Economics, Kardan University, Parwan-e- Du Square, Kabul, Afghanistan \\ Correspondence should be addressed to Saeed Gul; s.gul@kardan.edu.af
}

Received 31 August 2021; Revised 5 November 2021; Accepted 12 November 2021; Published 14 December 2021

Academic Editor: Naeem Jan

Copyright (C) 2021 Arif Mehmood et al. This is an open access article distributed under the Creative Commons Attribution License, which permits unrestricted use, distribution, and reproduction in any medium, provided the original work is properly cited.

\begin{abstract}
In this study, new operations of union, intersection, and complement are defined with the help of vague soft sets in a new way that is in both true and false statements, union is defined with maximum, and intersection is defined with minimum. On the basis of these operations, vague soft topology is defined. Pairwise vague soft open sets and pairwise vague soft closed sets are defined in vague soft bitopological structures (VSBTS). Moreover, generalized vague soft open sets are introduced in VSBTS concerning soft points of the space. On the basis of generalized vague soft open sets, separation axioms are also introduced. In continuation, these separations axioms are engaged with other important results in VSBTS.
\end{abstract}

\section{Introduction}

Fuzzy set theory [1] is the most importantly effective way to deal with vagueness and incomplete data, and it is being developed and used in various fields of science. Fuzzy set theory is the most attractive approach to address the uncertain situation. It is an entirely different approach in comparison to probability theory. It may be used in such situations where probability theory is getting failed. However, it has a shortcoming, i.e., it only addresses the membership value and is at a loss to address the nonmembership value. Atanassov [2] bridged the gap in fuzzy set theory with a new way and introduced the concept of intuitionistic fuzzy set theory. This approach supposes membership value and nonmembership value.

Molodtsov [3] shaked the pillars of set theory and installed the concept of soft set theory (SS) to address the uncertainty in a meaningful way. Soft set theory (SST) has a big hand as an application in many fields like function smoothness, Riemann integration, measurement theory, and game theory [4]. The concept of vague set theory which is generalization of fuzzy set theory was suggested by Gau and Buehrer [5]. A vague set is defined as truth-membership function and false membership function.

Wei et al. [6] combined the soft set and vague set structures and leaked out the concept of vague to soft set theory. Huang et al. [7] deeply studied [6] and pointed out some incorrect results. They verified the incorrect result with examples and gave some more new definitions. Wang and $\mathrm{Li}$ [8] initiated the concept of vague soft topological structures with title topological structure of vague soft sets. The authors discussed the basic concepts related to vague soft topological and studied the results in vague soft topology. Wang and $\mathrm{Li}$ [8] initiated the concept of vague soft topological structures with title topological structure of vague soft sets. They authors discussed the basic concepts related to vague soft topological and studied the results in vague soft topology. For better understanding, the authors provided number of examples. 
Mukherjee and Das [9] studied the concept of neutrosophic bipolar vague soft sets and some of its operations. It is the combination of neutrosophic bipolar vague sets and soft sets. Furthermore, the authors developed a decisionmaking method based on neutrosophic bipolar vague soft set. A numerical example has been shown. Some new operations on neutrosophic bipolar vague soft set have also been designed.

The organization of the study is as follows. We first attempted the fundamentals definitions of vague soft set including complement, subsets, equal sets, null set, absolute set, union, and intersection in a new approach. On the basis of these concepts, vague soft topological structure is defined that are useful for subsequent discussions. Al-Quran and Hassan [10] extended the notion of classical soft set to neutrosophic vague soft sets by applying the theory of soft sets to neutrosophic vague soft sets to make more effective results. They defined new operations. Finally, they applied the theory to decision-making problems. An ample of examples is provided to make the results more effective in this direction.

In our study, we worked with the new operations which are entirely different from references $[5,6]$. Then, unlike references $[7,8]$, vague soft topological structure is reconstructed. In Section 2, some basic operators are introduced in a new way. In Section 3, vague soft bitopology (VSBT) is addressed with ample of examples. Some results, union, and intersection are also studied (VSBT). In Section 4, some main results are addressed. In Section 5, main more results are addressed. In Section 6, some concluding remarks and future work are addressed.

\section{Preliminaries}

In this section, some fundamental concepts are addressed.

Definition 1. Let $\pi$ be key set (KS) and $\theta$ set of parameters. Let $\mathscr{L}(\pi)$ signifies power set of all vague sets on $\pi$. Then, a vague soft set $(\widetilde{\mathfrak{f}}, \theta)$ over $\pi$ is a set defined by a set-valued function $\tilde{\mathfrak{f}}$ representing a mapping $\widetilde{\mathfrak{f}}: \theta \longrightarrow \mathscr{L}(\pi)$, where $\widetilde{\mathfrak{f}}$ is called the approximate function of the vague soft set $(\widetilde{\mathfrak{f}}, \theta)$. It can be written as a set of ordered pairs: $(\widetilde{\mathfrak{f}}, \theta)=\left\{\left(\left(p, \kappa, \widetilde{F}_{\mathfrak{f}(n)^{(\kappa)}}, \mathbb{F}_{\widetilde{\mathfrak{f}}_{(n)}^{(\kappa)}}: \kappa \in \pi\right): p \in \theta\right\}\right.$.

Definition 2. Let $(\widetilde{\mathfrak{f}}, \theta)$ be a vague soft set over key set $\pi$. The complement of $(\widetilde{\mathfrak{f}}, \theta)$ is signified as $(\widetilde{\mathfrak{f}}, \theta)^{c}$ and is defined as

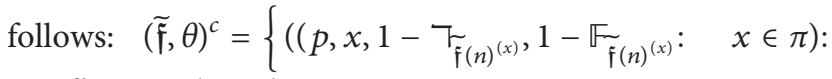
$p \in \theta\}$. It is clear that

$$
\left((\widetilde{\mathfrak{f}}, \theta)^{c}\right)^{c}=(\widetilde{\mathfrak{f}}, \theta)
$$

Definition 3. Let $(\widetilde{\mathfrak{f}}, \theta)$ and $(\widetilde{\rho}, \theta)$ be two vague soft subsets over key set $\pi$. $(\widetilde{\tilde{f}}, \theta)$ is supposed to be vague soft subset of $(\widetilde{\rho}, \theta)$ if $\tau_{\tilde{\mathfrak{f}}^{(n)}} \leqslant \tau_{\tilde{\rho}(n)^{(x)}}, \mathrm{F}_{\left.\widetilde{\mathfrak{f}}^{(n)}\right)^{(x)}} \leqslant \mathrm{F}_{\tilde{\rho}(n)^{(x)}}, \forall p \in \theta$, and $\forall x \in \pi$. It is signified as $(\widetilde{\mathfrak{f}}, \theta) \sqsubseteq(\widetilde{\rho}, \theta)$. $\cdot(\widetilde{\mathfrak{f}}, \theta)$ is said to be equal to $(\widetilde{\rho}, \theta)$ if $(\widetilde{\mathfrak{f}}, \theta)$ is a vague soft subset of $(\widetilde{\rho}, \theta)$ and $(\widetilde{\rho}, \theta)$ is a vague soft subset of $(\widetilde{\mathfrak{f}}, \theta)$. It is symbolized as $(\widetilde{\mathfrak{f}}, \theta)=(\widetilde{\rho}, \theta)$.

Definition 4. Let $\left(\widetilde{\mathfrak{f}}_{1}, \theta\right),\left(\widetilde{\mathfrak{f}}_{2}, \theta\right)$ be two vague soft subsets over key set $\pi$, so that $\left(\widetilde{\mathfrak{f}}_{1}, \theta\right) \neq\left(\widetilde{\mathfrak{f}}_{2}, \theta\right)$. Then, their union is signified as $\left(\widetilde{\mathfrak{f}}_{1}, \theta\right) \widetilde{\sqcup}\left(\widetilde{\mathfrak{f}}_{2}, \theta\right)=\left(\widetilde{\mathfrak{f}}_{3}, \theta\right)$ and is defined as $\left(\widetilde{\mathfrak{f}}_{3}, \theta\right)=\left\{\left(\left(p, x,{\widetilde{\mathfrak{f}_{3}}}_{(n)}{ }^{(k)}, \widetilde{F}_{\tilde{\mathfrak{F}}_{3}(n)}{ }_{(k)},: \kappa \in \pi\right): p \in \theta\right\}\right.$, where

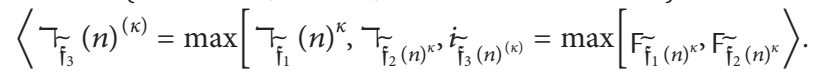

Definition 5. If $\left(\widetilde{\mathfrak{f}}_{1}, \theta\right),\left(\widetilde{\mathfrak{f}}_{2}, \theta\right)$ be two vague soft subsets over key set $\pi$, such that $\left(\widetilde{\mathfrak{f}}_{1}, \theta\right) \neq\left(\widetilde{\mathfrak{f}}_{2}, \theta\right)$. Then, their intersection is signified as $\left(\widetilde{\mathfrak{f}}_{1}, \theta\right) \widetilde{\Pi}\left(\widetilde{\mathfrak{f}}_{2}, \theta\right)=\left(\widetilde{\mathfrak{f}}_{3}, \theta\right)$ and is defined as $\left(\widetilde{\mathfrak{f}}_{3}, \theta\right)=\left\{\left(\left(p, x, \widetilde{\mathfrak{f}}_{\tilde{\mathfrak{F}}_{3}(n)}, \mathrm{F}_{\tilde{\mathfrak{f}}_{3}(n)},: \kappa \in \pi\right): p \in \theta\right\}\right.$, where

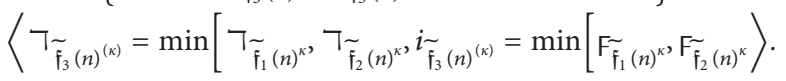

Definition 6. If $(\tilde{\mathfrak{f}}, \theta)$ be a vague soft set over key set, $\pi$ is said to be a null vague soft set (NVSS). If $\neg_{\mathfrak{f}(n)^{\kappa}}=0$, $\mathrm{F}_{\mathfrak{f}(n)^{\kappa}}=1 ; \forall n \in \theta, \forall \kappa \in \pi$.

It is signified as $0_{(\pi, \theta)}$.

Definition 7. If $(\widetilde{\tilde{f}}, \theta)$ be a vague soft over key set, $\pi$ is an absolute vague soft set if $\mathbb{T}_{\mathfrak{f}(n)^{\kappa}}=1, \mathbb{F}_{\mathfrak{f}(n)^{\kappa}}=0 ; \forall n \in \theta$, and $\forall \kappa \in \pi$.

Definition 8. If VSS $(\tilde{\pi}, \theta)$ be the family of all VS soft sets and $\tau \subset \operatorname{VSS}(\tilde{\pi}, \theta)$, then $\tau$ is said to be a VS soft topology on $\tilde{\pi}$ if $0_{(\pi, p)}, 1_{(\pi, p)}$ belong to $\tau$, the union of any number of VS soft sets in $\tau$ belong to $\tau$, and the intersection of a finite number of vague soft sets in $\tau$ belong to $\tau$. Then, $(\tilde{\pi}, \tau, \theta)$ is said to be a vague soft topology (VST) over $\tilde{\pi}$.

Definition 9. Let VSS $(\tilde{\pi})$ be the family of all vague soft sets over key set $\pi$. Then, $\left(\kappa_{\Delta_{1}, \Delta_{2}}\right)^{e}$ is called a vague soft point, for every $\in \tilde{\pi}, 0 \prec\left\{\Delta_{1}, \Delta_{2}\right\} \preccurlyeq 1, e \in \theta$, and is defined as follows:

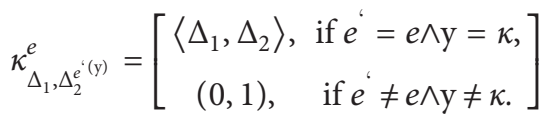

Definition 10. Let $(\widetilde{\mathfrak{f}}, \theta)$ be VSS over KS $\pi$. $\kappa_{\left(\Delta_{1}, \Delta_{2}\right)}^{e} \in \operatorname{NSS}(\tilde{\mathfrak{f}}, \theta)$ if

$$
\begin{aligned}
& \Delta_{1} \leqslant T_{\tilde{f}(n)^{\kappa}} \text {, } \\
& \Delta_{2} \preccurlyeq \mathrm{F}_{\mathfrak{f}(n)^{\kappa}} \text {. }
\end{aligned}
$$

Definition 11. $(\tilde{\pi}, \tau, \theta)$ be a vague soft topological space over $\pi$ and $(\widetilde{\mathfrak{f}}, \theta)$ be a vague soft over $\pi$. Vague soft set $(\widetilde{\mathfrak{f}}, \theta)$ in $(\pi, \tau, \theta)$ is called a vague soft nbhd of the vague soft point $\kappa_{\Delta_{1}, \Delta_{2}}^{\lambda} \in(\widetilde{\mathfrak{f}}, \theta)$, if there exists a NS open set $(\widetilde{\mathrm{g}}, \theta)$, such that $\kappa_{\Delta_{1}, \Delta_{2}}^{\lambda} \in(\widetilde{\mathrm{g}}, \theta)$. 


\section{Vague Soft Bitopological Structure}

In this part, the concept of vague soft bitopological space (VSBTS) is defined. Furthermore, new types of open and closed sets have been introduced in the same space. Example of vague soft bitopological space is given. Intersection of vague soft bitopological space is addressed. Union of two vague soft bitopological spaces need not be vague soft topological space. This situation is established with example. Pairwise vague soft open and soft closed sets are addressed in vague soft bitopological space. Some theorems related to this concept are also addressed.

Definition 12. If $\left\langle\pi, \tau_{1}, \theta\right\rangle$ and $\left\langle\pi, \tau_{1}, \theta\right\rangle$ are two VSTS, then $\left\langle\pi, \tau_{1}, \tau_{2}, \theta\right\rangle$ is called VSBTS. Let $\left\langle\pi, \tau_{1}, \tau_{2}, \theta\right\rangle$ be vague soft bitopological space. A vague soft set $(f, \theta)$ is said to be open in $\left\langle\pi, \tau_{1}, \tau_{2}, \theta\right\rangle$ if there exists a vague soft open set $\left(f_{1}, \theta\right)$ in $\tau_{1}$ and vague soft open set $\left(f_{2}, \theta\right)$ in $\tau_{2}$, such that $(f, \theta)=\left(f_{1}, \theta\right) \cup\left(f_{2}, \theta\right)$. The vague soft set $(f, \theta)$ is said to be close in $\left\langle\pi, \tau_{1}, \tau_{2}, \theta\right\rangle$ if its complement is open in $\left\langle\pi, \tau_{1}, \tau_{2}, \theta\right\rangle$.

Example 1. Let $\pi=\left\{\kappa_{1}, \kappa_{2}, \kappa_{3}\right\}, \theta=\left\{e_{1}, e_{2}\right\}, \tau_{1}=\left\{0_{(\pi, \theta)}\right.$, $\left.1_{(\pi, \theta)},\left(\omega_{1}, \theta\right),\left(\omega_{2}, \theta\right)\right\}$, and $\tau_{1}=\left\{0_{(\pi, \theta)}, 1_{(\pi, \theta)},\left(\wedge_{1}, \theta\right),\left(\wedge_{2}\right.\right.$, $\theta)\}$, where $\left(\omega_{1}, \theta\right),\left(\omega_{2}, \theta\right),\left(\kappa_{1}, \theta\right)$, and $\left(\kappa_{2}, \theta\right)$ being vague soft sets are as follows:

$$
\begin{aligned}
& f_{\left(\omega_{1}, \theta\right)}\left(e_{1}\right)=\left[\left\langle\kappa_{1}, \frac{02}{10}, \frac{03}{10}\right\rangle,\left\langle\kappa_{2}, \frac{04}{10}, \frac{04}{10}\right\rangle,\left\langle\kappa_{3}, \frac{02}{10}, \frac{04}{10}\right\rangle\right], \\
& f_{\left(\omega_{1}, \theta\right)}\left(e_{2}\right)=\left[\left\langle\kappa_{1}, \frac{03}{10}, \frac{02}{10}\right\rangle,\left\langle\kappa_{2}, \frac{01}{10}, \frac{05}{10}\right\rangle,\left\langle\kappa_{3}, \frac{04}{10}, \frac{03}{10}\right\rangle\right], \\
& f_{\left(\omega_{2}, \theta\right)}\left(e_{1}\right)=\left[\left\langle\kappa_{1}, \frac{04}{10}, \frac{03}{10}\right\rangle,\left\langle\kappa_{2}, \frac{04}{10}, \frac{05}{10}\right\rangle,\left\langle\kappa_{3}, \frac{03}{10}, \frac{05}{10}\right\rangle\right], \\
& f_{\left(\omega_{2}, \theta\right)}\left(e_{2}\right)=\left[\left\langle\kappa_{1}, \frac{03}{10}, \frac{04}{10}\right\rangle,\left\langle\kappa_{2}, \frac{02}{10}, \frac{06}{10}\right\rangle,\left\langle\kappa_{3}, \frac{04}{10}, \frac{06}{10}\right\rangle\right], \\
& f_{\left(\iota_{1}, \theta\right)}\left(e_{1}\right)=\left[\left\langle\kappa_{1}, \frac{05}{10}, \frac{04}{10}\right\rangle,\left\langle\kappa_{2}, \frac{06}{10}, \frac{06}{10}\right\rangle,\left\langle\kappa_{3}, \frac{04}{10}, \frac{06}{10}\right\rangle\right], \\
& f_{\left(\iota_{1}, \theta\right)}\left(e_{2}\right)=\left[\left\langle\kappa_{1}, \frac{04}{10}, \frac{06}{10}\right\rangle,\left\langle\kappa_{2}, \frac{03}{10}, \frac{07}{10}\right\rangle,\left\langle\kappa_{3}, \frac{05}{10}, \frac{07}{10}\right\rangle\right], \\
& f_{\left(\alpha_{2}, \theta\right)}\left(e_{1}\right)=\left[\left\langle\kappa_{1}, \frac{01}{10}, \frac{02}{10}\right\rangle,\left\langle\kappa_{2}, \frac{03}{10}, \frac{03}{10}\right\rangle,\left\langle\kappa_{3}, \frac{01}{10}, \frac{02}{10}\right\rangle\right], \\
& f_{\left(\kappa_{2}, \theta\right)}\left(e_{2}\right)=\left[\left\{\left\langle\kappa_{1}, \frac{01}{10}, \frac{02}{10}\right\rangle,\left\langle\kappa_{2}, \frac{03}{10}, \frac{03}{10}\right\rangle,\left\langle\kappa_{3}, \frac{01}{10}, \frac{02}{10}\right\rangle\right\}\right] .
\end{aligned}
$$

Then, $\quad\left(\omega_{1}, \theta\right) \cup\left(\omega_{2}, \theta\right)=\left(\omega_{2}, \theta\right), \quad\left(\omega_{1}, \theta\right) \cup\left(\iota_{1}, \theta\right)=$ $\left(\iota_{1}, \theta\right), \quad\left(\omega_{1}, \theta\right) \cup\left(\iota_{2}, \theta\right)=\left(\omega_{2}, \theta\right), \quad\left(\iota_{1}, \theta\right) \cup\left(\iota_{2}, \theta\right)=\left(\iota_{1}\right.$, $\theta),\left(\omega_{2}, \theta\right) \cup\left(\wedge_{2}, \theta\right)=\left(\omega_{2}, \theta\right)$ and $\left(\omega_{1}, \theta\right) \cap\left(\omega_{2}, \theta\right)=\left(\omega_{2}, \theta\right)$, $\left(\omega_{1}, \theta\right) \cap \curlywedge=\left(\iota_{1}, \theta\right), \quad\left(\omega_{1}, \theta\right) \cap\left(\iota_{2}, \theta\right)=\left(\omega_{2}, \theta\right), \quad\left(\iota_{1}, \theta\right) \cap$ $\left(\iota_{2}, \theta\right)=\left(\iota_{1}, \theta\right),\left(\omega_{2}, \theta\right) \cap\left(\iota_{2}, \theta\right)=\left(\iota_{2}, \theta\right)$.

Therefore, $\tau_{1}, \tau_{2}$ are VSTS on $\pi$, so $\left(\pi, \tau_{1}, \tau_{2}, \theta\right)$ is VSBTS.

Theorem 1. Let $\left(\pi, \tau_{1}, \tau_{2}, \theta\right)$ be a VSBTS. Then, $\tau_{1} \cap \tau_{2}$ is VSBTS on $\pi$.
Proof. (1) and (3) are obvious. For (2), we proceed as follows: let $\left\{\left(\omega_{i}, \theta\right) ; i \in I\right\} \in \tau_{1} \cap \tau_{2}$. Then, $\left(\omega_{i}, \theta\right) \in \tau_{1}$ and $\left(\omega_{i}, \theta\right) \in \tau_{2}$. Since $\tau_{2}, \tau_{2}$ are vague soft topologies on $\pi$, then $\cup_{i}\left(\omega_{i}, \theta\right) \in \tau_{1} \quad$ and $\quad \cup_{i}\left(\omega_{i}, \theta\right) \in \tau_{2} . \quad$ Therefore, $\cup_{i}\left(\omega_{i}, \theta\right) \in \tau_{1} \cap \tau_{2}$.

Remark 1. Let $\left(\pi, \tau_{1}, \tau_{2}, \theta\right)$ be VSBTS; then, $\tau_{1} \cup \tau_{2}$ need not be VSBTS on $\pi$.

Example 2. Let $\pi=\left\{\kappa_{1}, \kappa_{2}, \kappa_{3}\right\},=\left\{e_{1}, e_{2}\right\}, \quad \tau_{1}=\left\{0_{(\pi, \theta)}\right.$, $\left.1_{(\pi, \theta)},\left(\omega_{1}, \theta\right),\left(\omega_{2}, \theta\right),\left(\omega_{3}, \theta\right)\right\}, \quad$ and $\tau_{2}=\left\{0_{(\pi, \theta)}, 1_{(\pi, \theta)}\right.$, $\left(\kappa_{1}, \theta\right),\left(\iota_{2}, \theta\right)$, where $\left(\omega_{1}, \theta\right),\left(\omega_{2}, \theta\right),\left(\iota_{1}, \theta\right)$, and $\left(\iota_{2}, \theta\right)$ being vague soft sets are as follows:

$$
\begin{aligned}
& f_{\left(\omega_{1}, \theta\right)}\left(e_{1}\right)=\left[\left\langle\kappa_{1}, \frac{02}{10}, \frac{03}{10}\right\rangle,\left\langle\kappa_{2}, \frac{04}{10}, \frac{04}{10}\right\rangle,\left\langle\kappa_{3}, \frac{02}{10}, \frac{04}{10}\right\rangle\right], \\
& f_{\left(\omega_{1}, \theta\right)}\left(e_{2}\right)=\left[\left\langle\kappa_{1}, \frac{03}{10}, \frac{02}{10}\right\rangle,\left\langle\kappa_{2}, \frac{01}{10}, \frac{05}{10}\right\rangle,\left\langle\kappa_{3}, \frac{04}{10}, \frac{03}{10}\right\rangle\right], \\
& f_{\left(\omega_{2}, \theta\right)}\left(e_{1}\right)=\left[\left\langle\kappa_{1}, \frac{04}{10}, \frac{03}{10}\right\rangle,\left\langle\kappa_{2}, \frac{04}{10}, \frac{05}{10}\right\rangle,\left\langle\kappa_{3}, \frac{03}{10}, \frac{05}{10}\right\rangle\right], \\
& f_{\left(\omega_{2}, \theta\right)}\left(e_{2}\right)=\left[\left\langle\kappa_{1}, \frac{03}{10}, \frac{04}{10}\right\rangle,\left\langle\kappa_{2}, \frac{02}{10}, \frac{06}{10}\right\rangle,\left\langle\kappa_{3}, \frac{04}{10}, \frac{06}{10}\right\rangle\right], \\
& f_{\left(\omega_{3}, \theta\right)}\left(e_{1}\right)=\left[\left\langle\kappa_{1}, \frac{05}{10}, \frac{04}{10}\right\rangle,\left\langle\kappa_{2}, \frac{06}{10}, \frac{06}{10}\right\rangle,\left\langle\kappa_{3}, \frac{04}{10}, \frac{06}{10}\right\rangle\right], \\
& f_{\left(\omega_{3}, \theta\right)}\left(e_{2}\right)=\left[\left\langle\kappa_{1}, \frac{04}{10}, \frac{06}{10}\right\rangle,\left\langle\kappa_{2}, \frac{03}{10}, \frac{07}{10}\right\rangle,\left\langle\kappa_{3}, \frac{05}{10}, \frac{07}{10}\right\rangle\right], \\
& f_{\left(\kappa_{1}, \theta\right)}\left(e_{1}\right)=\left[\left\langle\kappa_{1}, \frac{05}{10}, \frac{04}{10}\right\rangle,\left\langle\kappa_{2}, \frac{06}{10}, \frac{06}{10}\right\rangle,\left\langle\kappa_{3}, \frac{04}{10}, \frac{06}{10}\right\rangle\right], \\
& f_{\left(\kappa_{2}, \theta\right)}\left(e_{1}\right)=\left[\left\langle\kappa_{1}, \frac{01}{10}, \frac{02}{10}\right\rangle,\left\langle\kappa_{2}, \frac{03}{10}, \frac{03}{10}\right\rangle,\left\langle\kappa_{3}, \frac{01}{10}, \frac{02}{10}\right\rangle\right], \\
& \left.f_{\left(\kappa_{1}, \theta\right)}\left(e_{2}\right)=\left[\left\langle\kappa_{1}, \frac{04}{10}, \frac{06}{10}\right\rangle,\left\langle\kappa_{2}, \frac{03}{10}, \frac{07}{10}\right\rangle,\left\langle\kappa_{3}, \frac{05}{10}, \frac{07}{10}\right\rangle\right],\left\langle\kappa_{2}, \frac{03}{10}, \frac{03}{10}\right\rangle,\left\langle\kappa_{3}, \frac{01}{10}, \frac{02}{10}\right\rangle\right] .
\end{aligned}
$$

Here, $\quad \tau_{1} \cup \tau_{2}=\left\{0_{(Y, \theta)}, 1_{(\pi, \theta)},\left(\omega_{1}, \theta\right),\left(\omega_{2}, \theta\right),\left(\omega_{3}, \theta\right)\right.$, $\left.\left(\iota_{1}, \theta\right),\left(\iota_{2}, \theta\right)\right\}$ is not a vague soft topology on $\pi$. Since, $\left(\omega_{3}, \theta\right) \cup\left(\wedge_{2}, \theta\right) \notin \tau_{1} \cup \tau_{2}$.

Definition 13. Let $\left(\pi, \tau_{1}, \tau_{2}, \theta\right)$ be VSBTS. Then, a vague soft set

$$
(\ltimes, \theta)=\left\{\left(e,\left\{\left\langle x, T_{\ltimes(e)}(x), F_{\ltimes(e)}(x)\right\rangle\right\}: \quad x \in \pi, e \in \theta\right\},\right.
$$

is called as a pairwise vague soft open set (PVSOS) if there exist a vague soft open set $\left(\ltimes_{1}, \theta\right)$ in $\tau_{1}$ and a vague soft open set $\left(\ltimes_{2}, \theta\right)$ in $\tau_{2}$, such that for all $x \in \pi$, 


$$
(\ltimes, \theta)=\left(\ltimes_{1}, \theta\right) \cup\left(\ltimes_{2}, \theta\right)=\left\{\left(e,\left\{\begin{array}{c}
\left\langle x, \max \left\{T_{\ltimes(e)}(x), T_{G(e)}(x)\right\},\right. \\
\left.\max \left\{F_{\ltimes(e)}(x), F_{G(e)}(x)\right\}\right\rangle
\end{array}\right\}\right): \quad e \in \theta\right\} .
$$

Definition 14. Let $\left(\pi, \tau_{1}, \tau_{2}, \theta\right)$ be VSBTS. Then, a vague soft set

$$
(\ltimes, \theta)=\left\{\left(e,\left\{\left\langle x, T_{\ltimes(e)}(x),, F_{\ltimes(e)}(x)\right\rangle\right\}\right): \quad x \in \pi, e \in \theta\right\}, \quad(10)
$$

is called as PVSOS if there exist a vague soft open set $\left(\ltimes_{1}, \theta\right)$ in $\tau_{1}$ and a vague soft open set $\left(\ltimes_{2}, \theta\right)$ in $\tau_{2}$, such that for all $x \in \pi$,

$$
(\ltimes, \theta)=\left(\ltimes_{1}, \theta\right) \cup\left(\ltimes_{2}, \theta\right)=\left\{\left(e,\left\{\begin{array}{c}
\left\langle x, \max \left\{T_{H(e)}(x), T_{G(e)}(x)\right\},\right. \\
\left.\max \left\{F_{\ltimes(e)}(x), F_{G(e)}(x)\right\}\right\rangle
\end{array}\right\}\right): \quad e \in \theta\right\} .
$$

The set of all pairwise vague open sets in $\left(\pi, \tau_{1}, \tau_{2}, \theta\right)$ is denoted by PVSO $\left(\pi, \tau_{1}, \tau_{2}, \theta\right)$.

Definition 15. Let $\left(\pi, \tau_{1}, \tau_{2}, \theta\right)$ be VSBTS. Then, a vague soft set

$$
(\ltimes, \theta)=\left\{\left(e,\left\{\left\langle x, T_{\ltimes(e)}(x), F_{\ltimes(e)}(x)\right\rangle\right\}\right): \quad x \in \pi, e \in \theta\right\},
$$

is called as a pairwise vague soft closed set (PVSC) if $(\ltimes, \theta)^{c}$ is PVSO. It is clear that $(\ltimes, \theta)$ is a PVSC set if there exist VSOC $\left(\ltimes_{1}, \theta\right)$ in $\tau_{1}$ and VSOC $\left(\ltimes_{2}, \theta\right)$ in $\tau_{2}$, such that for all $x \in \pi$,

$$
(\ltimes, \theta)=\left(\ltimes_{1}, \theta\right) \cap\left(\ltimes_{2}, \theta\right)=\left\{\left(e,\left\{\begin{array}{c}
\left\langle x, \min \left\{T \ltimes(x), T_{G(e)}(x)\right\},\right\rangle \\
\left.\min \left\{F_{\ltimes(e)}(x), F_{G(e)}(x)\right\}\right\rangle
\end{array}\right\}\right): \quad e \in \theta\right\} .
$$

The set of all PVSC in $\left(\pi, \tau_{1}, \tau_{2}, \theta\right)$ is denoted by Proof $\operatorname{PVSC}\left(\pi, \tau_{1}, \tau_{2}, \theta\right)$.

Theorem 2. Let $\left(\pi, \tau_{1}, \tau_{2}, \theta\right)$ be VSBTS. In this case,

(1) $0_{(\pi, \theta)}, 1_{(\pi, \theta)} \in \operatorname{PVSO}\left(X, \tau_{1}, \tau_{2}, \theta\right)$

(2) If $\left\{\left(\ltimes_{i}, \theta\right) \mid i \in I\right\} \subseteq P V S O\left(\pi, \tau_{1}, \tau_{2}, \theta\right)$, then $\cup_{i \in I}\left(\ltimes_{i}, \theta\right) \in \operatorname{PVSO}\left(\pi, \tau_{1}, \tau_{2}, \theta\right)$

(3) If $\left\{\left(G_{i}, \theta\right) \mid i \in I\right\} \subseteq P V S C\left(\pi, \tau_{1}, \tau_{2}, \theta\right)$, then $\cap_{i \in I}\left(G_{i}\right.$, $\theta) \in \operatorname{PVSC}\left(\pi, \tau_{1}, \tau_{2}, \theta\right)$
(1) Since $0_{(X, \theta)} \cup 0_{(\pi, \theta)}=0_{(\pi, \theta)}, \quad 1_{(\pi, \theta)} \cup 1_{(\pi, \theta)}=1_{(\pi, \theta)}$, then $0_{(\pi, \theta)}$ and $1_{(\pi, \theta)}$ are PVSC

(2) Since $\left(\ltimes_{i}, \theta\right) \in \operatorname{PVSO}\left(X, \tau_{1}, \tau_{2}, \theta\right)$, there exist $\left(\ltimes_{i}^{1}, \theta\right) \in \tau_{1}, \quad\left(\ltimes_{i}^{1}, \theta\right) \in \tau_{2}, \quad$ such that $\left(\ltimes_{i}, \theta\right)=$ $\left(\ltimes_{i}^{1}, \theta\right) \cup\left(\ltimes_{i}^{1}, \theta\right)$ for all $i \in I$. Then,

$$
\bigcup_{i \in I}\left(\ltimes_{i}, \theta\right)=\cup_{i \in I}\left(\left(\ltimes_{i}^{1}, \theta\right) \cup\left(\ltimes_{i}^{1}, \theta\right)\right)=\left(\cup_{i \in I}\left(\ltimes_{i}^{1}, \theta\right)\right) \cup\left(\cup_{i \in I}\left(\ltimes_{i}^{2}, \theta\right)\right) .
$$

As $\tau_{1}, \tau_{2}$ are VST on $\pi, \cup_{i \in I}\left(\ltimes_{i}^{1}, \theta\right) \in \tau_{1}$ and $\cup_{i \in I}\left(\ltimes_{i}^{2}, \theta\right) \in \tau_{1}$

Therefore, $\cup_{i \in I}\left(\ltimes_{i}, \theta\right) \in \operatorname{PNSO}\left(\pi, \tau_{1}, \tau_{2}, \theta\right)$
(3) Since $\left(G_{i}, \theta\right) \in \operatorname{PVSC}\left(X, \tau_{1}, \tau_{2}, \theta\right)$, there exist $\left(G_{i}^{1}, \theta\right)^{c} \in \tau_{1}$ and $\left(G_{i}^{2}, \theta\right)^{c} \in \tau_{2}$, such that $\left(G_{i}, \theta\right)=$ $\left(G_{i}^{1}, \theta\right) \cap\left(G_{i}^{2}, \theta\right)$ for all $i \in I$. Then,

$$
\bigcap_{i \in I}\left(G_{i}, \theta\right)=\bigcap_{i \in I}\left(\left(G_{i}^{1}, \theta\right) \cap\left(G_{i}^{2}, \theta\right)\right)=\left(\cap_{i \in I}\left(G_{i}^{1}, \theta\right)\right) \cap\left(\cap_{i \in I}\left(G_{i}^{2}, \theta\right)\right) .
$$

Then, $\cap_{i \in I}\left(G_{i}, \theta\right) \in \operatorname{PVSC}\left(\pi, \tau_{1}, \tau_{2}, \theta\right)$ as $\left(\cap_{i \in I}\left(G_{i}^{1}\right.\right.$, $\theta))^{c} \in \tau_{1}$, and $\left(\cap_{i \in I}\left(G_{i}^{2}, \theta\right)\right)^{c} \in \tau_{1}$.
Definition 16. Let $\left(\pi, \tau_{1}, \tau_{2}, \theta\right)$ be VSBTS, $(\wedge, \theta) \in \operatorname{VSS}(\pi)$. The PVS closure of $(<, \theta)$, denoted by $\operatorname{cl}_{P}^{\mathrm{VSS}}(<, \theta)$, is the intersection of all PVSC containing $(<, \theta)$, i.e., 


$$
\operatorname{cl}_{P}^{\mathrm{VSS}}(\wedge, \theta)=\cap\{(\omega, \theta) \in \operatorname{PVSC}(X) \mid(\wedge, \theta) \subset(\omega, \theta)\} .
$$

It is clear that $\mathrm{cl}_{P}^{\mathrm{VSS}}(\wedge, \theta)$ is the smallest PVSCS containing $(<, \theta)$.

Theorem 3. Let $\left(\pi, \tau_{1}, \tau_{2}, \theta\right)$ beVSBTS and $(\llcorner, \theta),(\omega, \theta) \in \operatorname{VSS}(\pi)$. Then,

(1) $c l_{P}^{V S S}\left(0_{(\pi, \theta)}\right)=0_{(\pi, \theta)}$ and $c l_{P}^{V S S}\left(1_{(\pi, \theta)}\right)=1_{(\pi, \theta)}$

(2) $(\wedge, \theta) \subseteq c l_{P}^{V S S}(\wedge, \theta)$

(3) $(<, \theta)$ is a PVSCS if $c_{P}^{V S S}(<, \theta)=(\llcorner, \theta)$

(4) $c l_{P}^{V S S}(\wedge, \theta) \subseteq c l_{P}^{V S S}(\omega, \theta)$ if $(<, \theta) \subseteq(\omega, \theta)$

(5) $c l_{P}^{V S S}(\wedge, \theta) \cup c l_{P}^{V S S}(\omega, \theta) \subset c l_{P}^{V S S}((<, \theta) \cup(\omega, \theta))$

(6) $c l_{P}^{V S S}\left(c l_{P}^{V S S}(\llcorner, \theta))=c l_{P}^{V S S}\left(\llcorner, \theta)\right.\right.$, i.e., $\quad c l_{P}^{V S S}(\llcorner, \theta)$ is PVSCS

Proof It is obvious.

Theorem 4. Let $\left(\pi, \tau_{1}, \tau_{2}, \theta\right)$ be VSBTS, $(\wedge, \theta) \in \operatorname{VSS}(\pi)$. Then, $x_{(\alpha, \beta)}^{e} \in \operatorname{cl}_{P}^{V S S}(\kappa, \theta)$ if and only if for all $U_{x_{(\alpha, \beta)}^{e}} \in \tau_{12}^{(\alpha, \beta}\left(x_{(\alpha, \beta)}^{e}\right)$, where $U_{x^{e}}$ is any PVSOS containing $x_{(\alpha, \beta)}^{e_{(\alpha, \beta)}}$, and $\tau_{12}\left(x_{(\alpha, \beta)}^{e}\right)$ is the family of all PVSOS containing $x_{(\alpha, \beta)}^{e}, U_{x_{(\alpha, \beta)}^{e}} \cap(<, \theta) \neq 0_{(\pi, \theta)}$.

Proof. Let $x_{(\alpha, \beta)}^{e} \in \mathrm{cl}_{P}^{\mathrm{VSS}}(\kappa, \theta)$, and suppose that there exists $U_{x_{(\alpha, \beta, \gamma)}^{e}} \in \tau_{12}\left(x_{(\alpha, \beta)}^{e}\right)$, such that $U_{x_{(\alpha, \beta)}^{e}} \cap(<, \theta)=0_{(\pi, \theta)}$. Then, $(\wedge, \theta) \subset\left(U_{x_{(\alpha, \beta)}^{e}}\right)^{c} . \quad$ Thus, $\quad \operatorname{cl}_{P}^{\mathrm{VSS}}(\wedge, \theta) \subset \operatorname{cl}_{P}^{\mathrm{VSS}}\left(U_{x_{(\alpha, \beta)}^{e}}\right)^{c}$ $=\left(U_{\left.x_{(\alpha, \beta)}^{e}\right)}\right)^{c}$, which implies $\operatorname{cl}_{P}^{\mathrm{VSS}}(\wedge, \theta) \cap U_{x_{(\alpha, \beta)}^{e}}=0_{(\pi, \theta)}$, a contradiction.

Conversely, $\quad x_{(\alpha, \beta)}^{e} \notin \mathrm{cl}_{P}^{\mathrm{NSS}}(\alpha, \theta)$; then, $x_{(\alpha, \beta)}^{e} \in\left(\mathrm{cl}_{P}^{\mathrm{VSS}}\right.$ $(\alpha, \theta))^{c} \in \tau_{12}\left(x_{(\alpha, \beta)}^{e}\right)$. Therefore, by hypothesis, $\left(\operatorname{cl}_{P}^{\mathrm{VSS}}(<, \theta)\right)^{c} \cap(<, \theta) \neq 0_{(\pi, \theta)}$, a contradiction.

\section{Main Results}

In this section, some new definitions are introduced in vague soft bitopological spaces. All these definitions are the backbone for the upcoming study. Among these definitions, definition (3) became source of motivation for our results which are discussed in this section and in the next section. All the results discussed in this section are discussed with respect to soft points of the spaces.

Definition 17. Let $\left\langle\pi, \tau_{1}, \tau_{2}, \theta\right\rangle$ be VSBTS over $\pi,(\widetilde{\mathfrak{f}}, \theta)$ be a VS set over $\pi$. Then, $(\widetilde{\mathfrak{f}}, \theta)$ is

(1) Vague soft semiopen if $(\widetilde{\mathfrak{f}}, \theta) \subseteq \operatorname{VScl}(\operatorname{VSint}(\widetilde{\mathfrak{f}}, \theta))$

(2) Vague soft preopen if $(\widetilde{\mathfrak{f}}, \theta) \subseteq \operatorname{VSint}(\operatorname{VScl}(\widetilde{\mathfrak{f}}, \theta))$

(3) Vague $\operatorname{soft}_{* \text { open if }}(\widetilde{\mathfrak{f}}, \theta) \subseteq \operatorname{VScl}(\operatorname{VSint}(\widetilde{\mathfrak{f}}, \theta)) \sqcup V S$ $\operatorname{int}(\operatorname{VScl}(\widetilde{\mathfrak{f}}, \theta))$ and vague soft $*_{b}$ close if $(\widetilde{\mathfrak{f}}, \theta) \supseteq \operatorname{VScl}(\operatorname{VSint}(\widetilde{\mathfrak{f}}, \theta)) \sqcap \operatorname{VSint}(\operatorname{VScl}(\widetilde{\mathfrak{f}}, \theta))$.

Definition 18. Let $\left\langle\pi, \tau_{1}, \tau_{2}, \theta\right\rangle$ be VSBTS over $\pi$, $\kappa_{1}^{\lambda}\left\langle\Delta_{1}, \Delta_{2}\right\rangle \neq \kappa_{2}^{\lambda^{\prime}}\left\langle\Delta_{1} /, \Delta_{2} /\right\rangle$ are VS points. If there exist VS $*_{b}$ open sets $(\widetilde{\mathfrak{f}}, \theta)$ and $(\widetilde{\mathrm{g}}, \theta)$, such that $\kappa_{1}^{\lambda}\left\langle\Delta_{1}, \Delta_{2}\right\rangle \in(\widetilde{\mathfrak{f}}, \theta), \kappa_{1}^{\lambda}\left\langle\Delta_{1}, \Delta_{2}\right\rangle \sqcap(\widetilde{\mathrm{g}}, \theta)=0_{(\langle\tilde{\pi}\rangle, \theta)}$, or $\kappa_{2}^{\lambda^{\prime}}\left\langle\Delta_{1} /\right.$, $\left.\Delta_{2} /\right\rangle \in(\widetilde{\mathrm{g}}, \theta), \kappa_{2}^{\lambda^{\prime}}\left\langle\Delta_{1} /, \Delta_{2} /\right\rangle \sqcap(\widetilde{\mathfrak{f}}, \theta)=0_{(\langle\tilde{\pi}\rangle, \theta) .}$ Then, $\left\langle\pi, \tau_{1}, \tau_{2}, \theta\right\rangle$ is called a NSB $*_{b 0}$ space.

Definition 19. Let $\left\langle\pi, \tau_{1}, \tau_{2}, \theta\right\rangle$ be VSBTS over $\widetilde{x}$, and $\kappa_{1}^{\lambda}\left\langle\Delta_{1}, \Delta_{2}\right\rangle \neq \kappa_{2}^{\lambda^{\prime}}\left\langle\Delta_{1} /, \Delta_{2} /\right\rangle$ are VS points. If there exist $\mathrm{VS} *_{b}$-open sets $(\widetilde{\mathfrak{f}}, \theta),(\widetilde{\mathrm{g}}, \theta)$, such that $\kappa_{1}^{\lambda}\left\langle\Delta_{1}, \Delta_{2}\right\rangle \in$ $(\widetilde{\mathfrak{f}}, \theta), \kappa_{1}^{\lambda}\left\langle\Delta_{1}, \Delta_{2}\right\rangle \sqcap(\widetilde{\mathrm{g}}, \theta)=0{ }_{(\langle\widetilde{\pi}\rangle, \theta)} \quad$ and $\quad \kappa_{2}^{\lambda^{\prime}}\left\langle\Delta_{1} /, \Delta_{2} /\right\rangle \epsilon$ $(\widetilde{\mathrm{g}}, \theta), \kappa_{2}^{\lambda^{\prime}}\left\langle\Delta_{1} /, \Delta_{2} /\right\rangle \sqcap(\widetilde{\mathfrak{f}}, \theta)=0_{(\langle\widetilde{\pi}\rangle, \theta)}$. Then, $\left\langle\pi, \tau_{1}, \tau_{2}, \theta\right\rangle$ is called a $\mathrm{VSB} *_{b 1}$ space.

Definition 20. Let $\left\langle\pi, \tau_{1}, \tau_{2}, \theta\right\rangle$ be VSBTS over $\pi, \kappa_{1}^{\lambda}\left\langle\Delta_{1}, \Delta_{2}\right\rangle \neq \kappa_{2}^{\lambda^{\prime}}\left\langle\Delta_{1} /, \Delta_{2} /\right\rangle$ are VS points. If $\exists \mathrm{VS} *_{b}$ open set $(\widetilde{\mathfrak{f}}, \theta), \quad(\widetilde{\mathrm{g}}, \theta), \quad$ such that $\kappa_{1}^{\lambda}\left\langle\Delta_{1}, \Delta_{2}\right\rangle \in(\widetilde{\mathfrak{f}}, \theta)$, $\kappa_{2}^{\lambda^{\prime}}\left\langle\Delta_{1} /, \Delta_{2} /\right\rangle \in(\widetilde{\mathrm{g}}, \theta)$, and $(\widetilde{\mathfrak{f}}, \theta) \sqcap(\widetilde{\mathrm{g}}, \theta)=0_{(\langle\widetilde{\pi}\rangle, \theta)}$. Then, $\left\langle\pi, \tau_{1}, \tau_{2}, \theta\right\rangle$ is called a VSB ${ }^{* *}{ }_{b 2}$ space.

Example 3. Let $\tilde{\pi}=\left\{\kappa_{1}, \kappa_{2}\right\}$, the set of conditions by $\theta=\left\{\lambda_{1}, \lambda_{2}\right\}$. Let us consider $\kappa_{1_{(0.1,0.4)}}^{\lambda_{1}}, \kappa_{1_{(0.2,0.5)}}^{\lambda_{2}}, \kappa_{2_{(0.30 .3)}}^{\lambda_{1}}$, and $\kappa_{2(0.4,4)}^{\lambda_{1}}$ be VS points. Then, the family $\tau_{1}=$

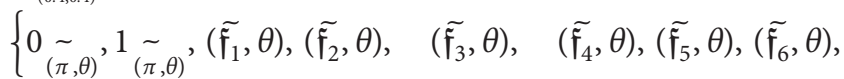
$\left(\tilde{\mathfrak{f}}_{7}, \theta\right),\left(\tilde{\mathfrak{f}}_{8}, \theta\right), \ldots, \quad\left\{\left(\widetilde{\mathfrak{f}_{15}}, \theta\right)\right.$, where $\left(\widetilde{\mathfrak{f}}_{1}, \theta\right)=\kappa_{1_{(0.1,0.4)}^{\lambda_{1}}},\left(\tilde{\mathfrak{f}}_{2}, \theta\right)$ $=\kappa_{1_{(0.20 .5)}^{\lambda_{2}},}, \quad\left(\widetilde{\mathfrak{f}}_{3}, \theta\right)=\kappa_{2(0.3,0.3)}^{\lambda_{1}},\left(\widetilde{\mathfrak{f}}_{4}, \quad \theta\right)=\kappa_{2(0.4,0.4)}^{\lambda_{2}}\left(\widetilde{\mathfrak{f}}_{5}, \theta\right)=\left(\tilde{\mathfrak{f}}_{1}\right.$, $\theta) \cup\left(\tilde{\mathfrak{f}}_{2}, \theta\right),\left(\tilde{\mathfrak{f}}_{6}, \theta\right)=\left(\tilde{\mathfrak{f}}_{1}, \theta\right) \cup\left(\tilde{\mathfrak{f}}_{3}, \theta\right), \quad\left(\tilde{\mathfrak{f}}_{7}, \theta\right)=\left(\tilde{\mathfrak{f}}_{2}, \theta\right) \cup\left(\tilde{\mathfrak{f}}_{4}\right.$, $\theta),\left(\widetilde{\mathfrak{f}}_{8}, \theta\right)=\left(\widetilde{\mathfrak{f}}_{2}, \theta\right) \cup\left(\widetilde{\mathfrak{f}}_{3}, \theta\right),\left(\widetilde{\mathfrak{f}_{9}}, \theta\right)=\left(\widetilde{\mathfrak{f}}_{2}, \theta\right) \cup\left(\widetilde{\mathfrak{f}}_{4}, \theta\right),\left(\widetilde{\tilde{f}_{10}}\right.$, $\theta)=\left(\tilde{\mathfrak{f}}_{3}, \theta\right) \cup\left(\tilde{\mathfrak{f}}_{4}, \theta\right),\left(\widetilde{\mathfrak{f}}_{11}, \theta\right)=\left(\tilde{\mathfrak{f}}_{1}, \theta\right) \cup\left(\tilde{\mathfrak{f}}_{2}, \theta\right) \cup\left(\tilde{\mathfrak{f}}_{3}, \theta\right),\left(\tilde{\mathfrak{f}}_{12}\right.$, $\theta)=\left(\widetilde{\mathfrak{f}_{1}}, \theta\right) \cup\left(\widetilde{\tilde{f}_{2}}, \theta\right) \cup\left(\widetilde{\tilde{f}_{4}}, \theta\right), \quad\left(\widetilde{\tilde{f}_{13}}, \theta\right)=\left(\widetilde{\mathfrak{f}_{2}}, \theta\right) \cup\left(\widetilde{\mathfrak{f}_{3}}, \theta\right) \cup$ $\left(\widetilde{\mathfrak{f}_{4}}, \theta\right),\left(\widetilde{\mathfrak{f}_{14}}, \theta\right)=\left(\tilde{\mathfrak{f}}_{1}, \theta\right) \cup\left(\tilde{\mathfrak{f}}_{3}, \theta\right) \cup\left(\tilde{\mathfrak{f}}_{4}, \theta\right), \quad\left(\widetilde{\mathfrak{f}_{15}}, \theta\right)=\left\{\kappa_{1_{(0,1,04)}^{\lambda_{1}}}\right.$, $\left.\kappa_{1_{(0,2,0.5)}^{\lambda_{2}}}^{\lambda_{2}}, \kappa_{2_{(0.3,0.3)}^{\lambda_{1}}}^{\lambda_{1}}, \kappa_{2_{(0.40,4)}^{\lambda_{2}}}\right\} \quad$ is VSTS. Also, $\tau_{2}=\left\{0_{(\langle\pi\rangle, \theta)}\right.$, $\left.1_{(\langle\tilde{\pi}\rangle, \theta)},\left(\widetilde{\mathfrak{f}_{1}}, \theta\right)\right\}$ VSTS. Thus, $\left(\pi, \tau_{1}, \tau_{2}, \theta\right)$ be VSBTS. Also, $\left(\pi, \tau_{1}, \tau_{2}, \theta\right)$ is $\mathrm{VSB} *_{b 2}$ structure.

Theorem 5. Let $\left\langle\pi, \tau_{1}, \tau_{2}, \theta\right\rangle$ be VSBTS. Then, $\left\langle\pi, \tau_{1}, \tau_{2}, \theta\right\rangle$ be a VSB* $*_{b 1}$ structure if and only if each VS point is $V S *_{b}$-closed.

Proof. Let $\left\langle\pi, \tau_{1}, \tau_{2}, \theta\right\rangle$ be VSBTS over and $\pi \cdot \kappa_{1}^{\lambda}\left\langle\Delta_{1}, \Delta_{2}\right\rangle$ be an arbitrary VS point. We establish $\kappa_{1}^{\lambda}\left\langle\Delta_{1}, \Delta_{2}\right\rangle$ is a $V$ soft $*_{b}$-open set. Let $\kappa_{2}^{\lambda^{\prime}}\left\langle\Delta_{1} /, \Delta_{2} /\right\rangle \in \kappa_{1}^{\lambda}\left\langle\Delta_{1}, \Delta_{2}\right\rangle$. Then, either $\kappa_{2}^{\lambda^{\prime}}\left\langle\Delta_{1} /, \Delta_{2} /\right\rangle>\kappa_{1}^{\lambda}\left\langle\Delta_{1}, \Delta_{2}\right\rangle$ or $\kappa_{2}^{\lambda^{\prime}}\left\langle\Delta_{1} /, \Delta_{2} /, \Delta_{3} /\right\rangle\left\langle\kappa_{2}^{\lambda^{\prime}}\left\langle\Delta_{1} /\right.\right.$, $\left.\Delta_{2} /\right\rangle$ or $\left.\quad \kappa_{2}^{\lambda^{\prime}}\left\langle\Delta_{1} /, \Delta_{2} /\right\rangle\right\rangle>\kappa_{1}^{\lambda}\left\langle\Delta_{1}, \Delta_{2}\right\rangle$ or $\kappa_{2}^{\lambda^{\prime}}\left\langle\Delta_{1} /, \Delta_{2} /\right\rangle<<$ $\kappa_{2}^{\lambda^{\prime}}\left\langle\Delta_{1} /, \Delta_{2} /\right\rangle$. This means that $\kappa_{2}^{\lambda^{\prime}}\left\langle\Delta_{1} /, \Delta_{2} /\right\rangle$ and $\kappa_{1}^{\lambda}\left\langle\Delta_{1}, \Delta_{2}\right\rangle$ are two distinct VS points. Thus, $\kappa_{1}>\kappa_{2}$ or $\kappa_{1}\left\langle\kappa_{2}\right.$ or $\lambda^{\prime}>\lambda$ or $\lambda^{\prime}\left\langle\lambda\right.$ or $\kappa_{1}>>\kappa_{2}$ or $\kappa_{1}\left\langle<\kappa_{2}\right.$ or $\lambda^{\prime}>>\lambda$ or $\lambda^{\prime}\langle<\lambda$. Since $(\tilde{\pi}, \tau, \theta)$ be a VS $*_{b 1}$ structure, there exists a VS $*_{b}$-open set $(\widetilde{\mathrm{g}}, \theta)$, so that $\kappa_{2}^{\lambda^{\prime}}\left\langle\Delta_{1} /, \Delta_{2} /\right\rangle \in(\widetilde{\mathrm{g}}, \theta)$ and $\kappa_{1}^{\lambda}\left\langle\Delta_{1}, \Delta_{2}\right\rangle \sqcap(\widetilde{\mathrm{g}}, \theta)=0_{(\langle\widetilde{\pi}\rangle, \theta)}$. Since, $\quad \kappa_{1}^{\lambda}\left\langle\Delta_{1}, \Delta_{2}\right\rangle \sqcap(\widetilde{\mathrm{g}}, \theta)=0_{(\langle\tilde{\pi}\rangle, \theta)}$. So, $\quad \kappa_{2}^{\lambda^{\prime}}\left\langle\Delta_{1} /, \Delta_{2} /\right\rangle \in$ 
$(\widetilde{\mathrm{g}}, \theta) \subset \kappa_{1}^{\lambda}\left\langle\Delta_{1}, \Delta_{2}\right\rangle$. Thus, $\kappa_{1}^{\lambda}\left\langle\Delta_{1}, \Delta_{2}\right\rangle$ is a VS $*_{b}$-open set, i.e., $\kappa_{1}^{\lambda}\left\langle\Delta_{1}, \Delta_{2}\right\rangle$ is a VS $*_{b}$-closed set. Suppose that each VS point $\kappa_{1}^{\lambda}\left\langle\Delta_{1}, \Delta_{2}\right\rangle$ is VS*b-closed. Then, $\left(\kappa_{1}^{\lambda}\left\langle\Delta_{1}, \Delta_{2}\right\rangle\right)^{c}$ is a $\mathrm{VS} *_{b}$-open set. Let $\kappa_{1}^{\lambda}\left\langle\Delta_{1}, \Delta_{2}\right\rangle \sqcap \kappa_{2}^{\lambda^{\prime}}\left\langle\Delta_{1} /, \Delta_{2} /\right\rangle=0_{(\langle\tilde{\pi}\rangle, \theta)}$. Thus, $\quad\left(\kappa_{2}^{\lambda^{\prime}}\left\langle\Delta_{1} /, \Delta_{2} /\right\rangle, \theta\right) \in\left(\kappa_{1}^{\lambda}\left\langle\Delta_{1}, \Delta_{2}\right\rangle\right)^{c}$, and $\kappa_{1}^{\lambda}\left\langle\Delta_{1}\right.$, $\left.\Delta_{2}\right\rangle \sqcap\left(\kappa_{1}^{\lambda}\left\langle\Delta_{1}, \Delta_{2}\right\rangle\right)^{c}=0_{(\langle\tilde{\pi}\rangle, \theta)}$. So, $\left\langle\pi, \tau_{1}, \tau_{2}, \theta\right\rangle$ be a VSB- $*_{b 1}$ space.

Theorem 6. Let $\left\langle\pi, \tau_{1}, \tau_{2}, \theta\right\rangle$ be VSTBS over the father set $\pi$. Then, $\left\langle\pi, \tau_{1}, \tau_{2}, \theta\right\rangle$ is VS $*_{b 2}$ space if and only if for distinct VS points $\kappa_{1}^{\lambda}\left\langle\Delta_{1}, \Delta_{2}\right\rangle, \kappa_{2}^{\lambda^{\prime}}\left\langle\Delta_{1} /, \Delta_{2} /\right\rangle$, there exists a VS* $*_{\text {-open }}$ set $(\widetilde{\mathfrak{f}}, \theta)$, containing but not $\kappa_{2}^{\lambda^{\prime}}\left\langle\Delta_{1} /, \Delta_{2} /\right\rangle$, such that $\kappa_{2}^{\lambda^{\prime}}\left\langle\Delta_{1} /, \Delta_{2} /\right\rangle \notin \overline{(\widetilde{\mathfrak{f}}, \theta)}$.

Proof. Let $\left.\kappa_{1}^{\lambda}\left\langle\Delta_{1}, \Delta_{2}\right\rangle\right\rangle \kappa_{2}^{\lambda^{\prime}}\left\langle\Delta_{1} /, \Delta_{2} /\right\rangle$ be two VS points in $\mathrm{VS} *_{b 2}$ space. Then, there exists disjoint VS*b open sets $(\widetilde{\mathfrak{f}}, \theta),(\widetilde{\mathrm{g}}, \theta)$, such that $\kappa_{1}^{\lambda}\left\langle\Delta_{1}, \Delta_{2}\right\rangle \in(\widetilde{\mathfrak{f}}, \theta)$ and $\kappa_{2}^{\lambda^{\prime}}\left\langle\Delta_{1} /\right.$, $\left.\Delta_{2} /\right\rangle \in(\widetilde{\mathrm{g}}, \theta)$. Since $\quad \kappa_{1}^{\lambda}\left\langle\Delta_{1}, \Delta_{2}\right\rangle, \theta \sqcap \kappa_{2}^{\lambda^{\prime}}\left\langle\Delta_{1} /, \Delta_{2} /\right\rangle=$ $0_{(\langle\tilde{\pi}\rangle, \theta)}$, and $(\tilde{\mathfrak{f}}, \theta) \sqcap(\widetilde{\mathrm{g}}, \theta)=0_{(\langle\tilde{\pi}\rangle, \theta)}, \cdot \kappa_{2}^{\lambda^{\prime}}\left\langle\Delta_{1} /, \Delta_{2} /\right\rangle \notin(\tilde{\mathfrak{f}}, \theta)$ implies $\quad \kappa_{2}^{\lambda^{\prime}}\left\langle\Delta_{1} /, \Delta_{2} /\right\rangle \notin \overline{(\widetilde{\mathfrak{f}}, \theta)}$. Next, suppose that $\kappa_{1}^{\lambda}\left\langle\Delta_{1}, \Delta_{2}\right\rangle>\kappa_{2}^{\lambda^{\prime}}\left\langle\Delta_{1} /, \Delta_{2} /\right\rangle$, there exists a VS $*_{b}$-open set $(\widetilde{\mathfrak{f}}, \theta)$ containing $\kappa_{1}^{\lambda}\left\langle\Delta_{1}, \Delta_{2}\right\rangle$ but not $\kappa_{2}^{\lambda^{\prime}}\left\langle\Delta_{1} /, \Delta_{2} /\right\rangle$, such that $\left.\kappa_{2}^{\lambda^{\prime}}\left\langle\Delta_{1} /, \Delta_{2} /\right\rangle \notin \overline{(\widetilde{\mathfrak{f}}, \theta)}\right)^{c}$, that is, $(\tilde{\mathfrak{f}}, \theta)$ and $\overline{(\tilde{\mathfrak{f}}, \theta)}{ }^{c}$ are mutually exclusive VS $*_{b}$-open sets supposing $\kappa_{1}^{\lambda}\left\langle\Delta_{1}, \Delta_{2}\right\rangle$ and $\kappa_{2}^{\lambda^{\prime}}\left\langle\Delta_{1} /, \Delta_{2} /\right\rangle$ in turn.

Theorem 7. Let $\left\langle\pi, \tau_{1}, \tau_{2}, \theta\right\rangle$ be VSBTS. Then, $\left\langle\pi, \tau_{1}, \tau_{2}, \theta\right\rangle$ is $V S *_{b 1}$ space if every VS point $\kappa_{1}^{\lambda}\left\langle\Delta_{1}, \Delta_{2}\right\rangle \in(\widetilde{\mathfrak{f}}, \theta) \in(\pi, \tau, \theta)$, if there exists a VS * ${ }_{b}$ open set $(\widetilde{\mathfrak{g}}, \theta)$, such that $\kappa_{1}^{\lambda}\left\langle\Delta_{1}, \Delta_{2}\right\rangle \in(\widetilde{\mathfrak{g}}, \theta) \subset \overline{(\widetilde{\mathfrak{g}}, \theta)} \subset(\widetilde{\mathfrak{f}}, \theta)$. Then, $\left\langle\pi, \tau_{1}, \tau_{2}, \theta\right\rangle$ is a $V S *_{b 2}$ space.

Proof. Suppose $\kappa_{1}^{\lambda}\left\langle\Delta_{1}, \Delta_{2}, \Delta_{3}\right\rangle \sqcap \kappa_{2}^{\lambda^{\prime}}\left\langle\Delta_{1} /, \Delta_{2} /\right\rangle=0{ }_{(\tilde{X}, \theta)}$. Since, $\left\langle\pi, \tau_{1}, \tau_{2}, \theta\right\rangle$ is VS $*_{b 1}$ space. $\kappa_{1}^{\lambda}\left\langle\Delta_{1}, \Delta_{2}\right\rangle$ and $\kappa_{2}^{\lambda^{\prime}}\left\langle\Delta_{1} /, \Delta_{2} /\right\rangle$ are the VS* $*_{b}$-closed sets in $\left\langle\pi, \tau_{1}, \tau_{2}, \theta\right\rangle$. Then, $\kappa_{1}^{\lambda}\left\langle\Delta_{1}, \Delta_{2}\right\rangle \in\left(\kappa_{2}^{\lambda^{\prime}}\left\langle\Delta_{1} /, \Delta_{2} /\right\rangle\right)^{c} \in\left\langle\pi, \tau_{1}, \tau_{2}, \theta\right\rangle$. Thus, there exists a VS* $*_{b}$-open set $(\tilde{g}, \theta) \in\left\langle\pi, \tau_{1}, \tau_{2}, \theta\right\rangle$, such that $\kappa_{1}^{\lambda}\left\langle\Delta_{1}, \Delta_{2}\right\rangle \in(\tilde{g}, \theta) \subset \overline{(\widetilde{g}, \theta)} \subset\left(\kappa_{2}^{\lambda^{\prime}}\left\langle\Delta_{1} /, \Delta_{2} /\right\rangle\right)^{c}$. So, we have $\kappa_{2}^{\lambda^{\prime}}\left\langle\Delta_{1} /, \Delta_{2} /\right\rangle \in(\tilde{g}, \theta)$ and $(\tilde{g}, \theta) \sqcap((\tilde{g}, \theta))^{c}=0_{(\langle\tilde{\pi}\rangle, \theta)}$, that is, $\left\langle\pi, \tau_{1}, \tau_{2}, \theta\right\rangle$ is a VS soft $*_{b 2}$ space.

Definition 21. Let $V$ be VSBTS. $(\widetilde{\mathfrak{f}}, \theta)$ be a VS $*_{b}$-closed set, and $\kappa_{1}^{\lambda}\left\langle\Delta_{1}, \Delta_{2}, \Delta_{3}\right\rangle \sqcap(\widetilde{\mathfrak{f}}, \theta)=0_{(\langle\tilde{\pi}\rangle, \theta)}$. If there exists VS $*_{b}$-open sets $\left(\tilde{g}_{1}, \theta\right)$ and $\left(\tilde{g}_{2}, \theta\right)$, such that $\kappa_{1}^{\lambda}\left\langle\Delta_{1}, \Delta_{2}\right\rangle \in\left(\tilde{g}_{1}, \theta\right),(\widetilde{\tilde{f}}, \theta) \subset\left(\tilde{g}_{2}, \theta\right)$, and $\kappa_{1}^{\lambda}\left\langle\Delta_{1}, \Delta_{2}\right\rangle \sqcap\left(\tilde{g}_{1}, \theta\right)$ $=0_{(\langle\tilde{\pi}\rangle, \theta)}$, then $\left\langle\pi, \tau_{1}, \tau_{2}, \theta\right\rangle$ is called a VBS $*_{b}$-regular space. $\left\langle\pi, \tau_{1}, \tau_{2}, \theta\right\rangle$ is said to be VS $*_{b 3}$ space, if it is both a VS regular and $\mathrm{VSB} *_{b 1}$ space.

Theorem 8. Let $\left\langle\pi, \tau_{1}, \tau_{2}, \theta\right\rangle$ be VSBTS. $\left\langle\pi, \tau_{1}, \tau_{2}, \theta\right\rangle$ is soft $*_{b 3}$ space if and only if for every $\kappa_{1}^{\lambda}\left\langle\Delta_{1}, \Delta_{2}\right\rangle \in(\widetilde{\tilde{f}}, \theta)$, that is, $(\tilde{g}, \theta) \in\left(\pi, \tau_{1}, \tau_{2}, \theta\right) \quad\left(\right.$ such $\quad$ that $\quad \kappa_{1}^{\lambda}\left\langle\Delta_{1}, \Delta_{2}\right\rangle \in(\tilde{g}, \theta)$ $\subset \overline{(\widetilde{g}, \theta)} \subset(\widetilde{\mathfrak{f}}, \theta)$.

Proof. Let $\left\langle\pi, \tau_{1}, \tau_{2}, \theta\right\rangle$ is $\mathrm{VSB} *_{b 3}$ space and $\kappa_{1}^{\lambda}\left\langle\Delta_{1}, \Delta_{2}\right\rangle \in(\widetilde{\mathfrak{f}}, \theta) \in\left\langle\pi, \tau_{1}, \tau_{2}, \theta\right\rangle$. Since $\left\langle\pi, \tau_{1}, \tau_{2}, \theta\right\rangle$ is $\mathrm{VS} *_{b 3}$ space for the VS point $\kappa_{1}^{\lambda}\left\langle\Delta_{1}, \Delta_{2}\right\rangle$, and VS $*_{b}$ is a closed set $(\widetilde{\mathfrak{f}}, \theta)^{c}, \exists\left(\tilde{g}_{1}, \theta\right)$ and $\left(\tilde{g}_{2}, \theta\right)$, such that $\kappa_{1}^{\lambda}\left\langle\Delta_{1}, \Delta_{2}\right\rangle \in\left(\tilde{g}_{1}, \theta\right),(\widetilde{\mathfrak{f}}, \theta)^{c} \subset\left(\tilde{g}_{2}, \theta\right)$, and $\left(\tilde{g}_{1}, \theta\right) \cap\left(\tilde{g}_{2}\right.$, $\theta)=0_{(\langle\tilde{x}\rangle, \theta)}$. Then, we have $\kappa_{1}^{\lambda}\left\langle\Delta_{1}, \Delta_{2}\right\rangle, \theta \in\left(\widetilde{g}_{1}, \theta\right) \subset\left(\widetilde{g}_{2}\right.$, $\theta)^{c} \subset(\tilde{\mathfrak{f}}, \theta)$. Since $\left(\tilde{g}_{2}, \theta\right)^{c} \mathrm{VS} *_{b}$ is a closed set, $\overline{\left(\tilde{g}_{1}, \theta\right)}$ $\subset\left(\tilde{g}_{2}, \theta\right)^{c}$. Conversely, let $\left.\kappa_{1}^{\lambda}\left\langle\Delta_{1}, \Delta_{2}\right\rangle, \theta \cap(\tilde{\mathscr{H}}, \theta)=0,\langle\tilde{x}\rangle, \theta\right)$ and $(\tilde{\mathscr{H}}, \theta)$ be a VS $*_{b}$ closed set. $\kappa_{1}^{\lambda}\left\langle\Delta_{1}, \Delta_{2}\right\rangle \in(\tilde{\mathscr{H}}, \theta)^{c}$, and from the condition of the theorem, we have $\kappa_{1}^{\lambda}\left\langle\Delta_{1}, \Delta_{2}\right\rangle \in(\tilde{g}, \theta) \subset \overline{(\widetilde{g}, \theta)} \subset(\tilde{\mathscr{H}}, \theta)^{c}$. Thus, $\kappa_{1}^{\lambda}\left\langle\Delta_{1}, \Delta_{2}\right\rangle \in$ $(\tilde{g}, \theta),(\widetilde{\mathscr{H}}, \theta) \subset \overline{(\tilde{g}, \theta)}^{c}$ and $(\tilde{g}, \theta) \cap \overline{(\tilde{g}, \theta)}^{c}=0_{(\langle\tilde{\pi}\rangle, \theta)}$. So, $\left\langle\pi, \tau_{1}, \tau_{2}, \theta\right\rangle$ is VSB $*_{b 3}$ space.

\section{More Main Results}

In this section, more main results are addressed. These results are discussed between two structures. The behavior of one structure can safely be transferred from one space into another space through soft functions by putting some conditions on the soft function. Similarly, through soft function, the engagement between structures and soft closed sets can be studied. The engagement is between second countable and soft limit points. The study of B. V. property, soft vague compactness, sequentially compactness, and countably compactness results are addressed with respect to soft points of the spaces.

Theorem 9. Let $\left\langle\pi, \tau_{1}, \tau_{2}, \theta\right\rangle$ be VSBTS, such that it is VSB*b Hausdorff space and $\left(\widetilde{\langle Y\rangle}, \mathfrak{F}_{1}, \mathfrak{F}_{2}, \theta\right)$ be another VSBTS. Let $\langle\mathrm{f}, \theta\rangle: \quad\left(\pi, \tau_{1}, \tau_{2}, \theta\right) \longrightarrow$ and $\left(\overline{\langle Y\rangle}, \mathfrak{F}_{1}, \mathfrak{F}_{2}, \theta\right)$ be another VSBTS which is a soft function, such that it is soft monotone and continuous. Then, $\left(\overline{\langle Y\rangle}, \mathfrak{F}_{1}, \mathfrak{F}_{2}, \theta\right)$ is also the characteristics of $V S B *_{b}$ Hausdorfness.

Proof. Suppose $\kappa_{1}^{\lambda}\left\langle\Delta_{1}, \Delta_{2}\right\rangle_{1}, \kappa_{1}^{\lambda}\left\langle\Delta_{1}, \Delta_{2}\right\rangle_{2} \in \tilde{\pi}$, such that either $\left.\kappa_{1}^{\lambda}\left\langle\Delta_{1}, \Delta_{2}\right\rangle{ }_{1}\right\rangle \kappa_{1}^{\lambda}\left\langle\Delta_{1}, \Delta_{2}\right\rangle_{2}$ or $\kappa_{1}^{\lambda}\left\langle\Delta_{1}, \Delta_{2}\right\rangle_{1} \prec \kappa_{1}^{\lambda}\left\langle\Delta_{1}, \Delta_{2}\right\rangle_{2}$. Since, $\langle\mathrm{f}, \theta\rangle$ is soft monotone. Let us suppose the monotonically increasing case. So, $\left.\left.\kappa_{1}^{\lambda}\left\langle\Delta_{1}, \Delta_{2}\right\rangle\right\rangle_{1}\right\rangle \kappa_{1}^{\lambda}\left\langle\Delta_{1}, \Delta_{2}\right\rangle_{2}$ or $\kappa_{1}^{\lambda}\left\langle\Delta_{1}, \Delta_{2}\right\rangle_{1} \prec \kappa_{1}^{\lambda}\left\langle\Delta_{1}, \Delta_{2}\right\rangle_{2}$ imply that $f_{\left.\kappa_{1}^{\lambda}\left\langle\Delta_{1}, \Delta_{2}\right\rangle_{1}\right\rangle}$ $f_{\kappa_{1}^{\lambda}\left\langle\Delta_{1}, \Delta_{2}\right\rangle_{2}}$ or $f_{\kappa_{1}^{\lambda}\left\langle\Delta_{1}, \Delta_{2}\right\rangle_{1}} \prec f_{\kappa_{1}^{\lambda}\left\langle\Delta_{1}, \Delta_{2}\right\rangle_{2}}$, respectively. Suppose $\kappa_{2}^{\lambda^{\prime}}\left\langle\Delta_{1} /, \Delta_{2} /\right\rangle_{1}, \kappa_{2}^{\lambda^{\prime}}\left\langle\Delta_{1} /, \Delta_{2} /\right\rangle_{2} \in \tilde{Y}$, such that $\left.\kappa_{2}^{\lambda^{\prime}}\left\langle\Delta_{1} /, \Delta_{2} /\right\rangle_{1}\right\rangle$ $\kappa_{2}^{\lambda^{\prime}}\left\langle\Delta_{1} /, \Delta_{2} /\right\rangle_{2}$ or $\kappa_{2}^{\lambda^{\prime}}\left\langle\Delta_{1} /, \Delta_{2} /\right\rangle_{1}\left\langle\kappa_{2}^{\lambda^{\prime}}\left\langle\Delta_{1} /, \Delta_{2} /\right\rangle_{2}\right.$; so, $\kappa_{2}^{\lambda^{\prime}}\left\langle\Delta_{1} /\right.$, $\left.\left.\Delta_{2} /\right\rangle_{1}\right\rangle \kappa_{2}^{\lambda^{\prime}}\left\langle\Delta_{1} /, \Delta_{2} /\right\rangle_{2}$ or $\kappa_{2}^{\lambda^{\prime}}\left\langle\Delta_{1} /, \Delta_{2} /\right\rangle_{1} \prec \kappa_{2}^{\lambda^{\prime}}\left\langle\Delta_{1} /, \Delta_{2} /\right\rangle_{2}$, respectively, such that $\kappa_{2}^{\lambda^{\prime}}\left\langle\Delta_{1} /, \Delta_{2} /\right\rangle_{1}=f_{\kappa_{1}^{\lambda}\left\langle\Delta_{1}, \Delta_{2}\right\rangle_{1}}, \kappa_{2}^{\lambda^{\prime}}$ $\left\langle\Delta_{1} /, \Delta_{2} /\right\rangle_{2}=f_{\kappa_{1}^{\lambda}\left\langle\Delta_{1}, \Delta_{2}\right\rangle_{2}}$. Since, $\left(\pi, \tau_{1}, \tau_{2}, \theta\right)$ is VSB $*_{b}$ Hausdorff space, so there exist mutually disjoint VS $*_{b}$ open sets $\left\langle\mathrm{k}_{1}, \theta\right\rangle$ and $\left\langle k_{2}, \theta\right\rangle \in\left(\pi, \tau_{1}, \quad \tau_{2}, \theta\right) \Rightarrow f\left(\left\langle k_{1}, \theta\right\rangle\right)$ and $f\left(\left\langle k_{2}, \theta\right\rangle\right) \in(\langle\widetilde{Y}\rangle, \mathfrak{F}, \theta)$. We claim that $f\left(\left\langle f_{1}, \theta\right\rangle\right) \widetilde{\cap} f\left(\left\langle k_{2}, \theta\right\rangle\right)=\widetilde{0_{(\widetilde{\pi}, \theta)}}$. Otherwise, $f\left(\left\langle k_{1}, \theta\right\rangle\right) \widetilde{\cap}$ 
$f\left(\left\langle k_{2}, \theta\right\rangle\right) \neq \widetilde{0_{(\widetilde{\pi}, \theta)}}$. Suppose there exists $\kappa_{3}^{\lambda \prime^{\prime}}\left\langle\Delta_{1}^{\prime \prime}, \Delta_{2}^{\prime \prime}, \Delta_{3}^{\prime \prime}\right\rangle_{1}$ $\in f\left(\left\langle k_{1},\right\rangle \theta\right) \widetilde{\cap} f\left(\left\langle k_{2}, \theta\right\rangle\right)$, there exists $\kappa_{3}^{\lambda \prime^{\prime}}\left\langle\Delta_{1}^{\prime \prime}, \Delta_{2}^{\prime \prime}\right\rangle_{1} \epsilon$ $f\left(\left\langle k_{1},\right\rangle \theta\right)$ and $\kappa_{3}^{\lambda \prime^{\prime}}\left\langle\Delta_{1}^{\prime \prime}, \Delta_{2}^{\prime \prime}\right\rangle_{1} \in f\left(\left\langle k_{2},\right\rangle \theta\right) . \kappa_{3}^{\lambda \prime^{\prime}}\left\langle\Delta_{1}^{\prime \prime}, \Delta_{2}^{\prime \prime}\right\rangle_{1} \epsilon$ $f\left(\left\langle k_{1}, \theta\right\rangle\right), f$ is soft one-one, there exists $\kappa_{3}^{\lambda \prime^{\prime}}\left\langle\Delta_{1}^{\prime \prime}, \Delta_{2}^{\prime \prime}\right\rangle_{2} \in\left\langle k_{1}, \theta\right\rangle$, such that $\kappa_{3}^{\lambda /}\left\langle\Delta_{1}^{\prime \prime}, \Delta_{2}^{\prime \prime}\right\rangle_{1}$ $=f\left(\kappa_{3}^{\lambda \prime^{\prime}}\left\langle\Delta_{1}^{\prime \prime}, \Delta_{2}^{\prime \prime}\right\rangle_{2}\right), \kappa_{3}^{\lambda \prime^{\prime}}\left\langle\Delta_{1}^{\prime \prime}, \Delta_{2}^{\prime \prime}\right\rangle_{1} \in f\left(\left\langle k_{2}, \theta\right\rangle\right)$ imply that there exists $\kappa_{3}^{\lambda^{\prime}}\left\langle\Delta_{1}^{\prime \prime}, \Delta_{2}^{\prime \prime}\right\rangle_{3} \in\left\langle k_{2}, \theta\right\rangle$, such that $f\left(\kappa_{3}^{\lambda \prime^{\prime}}\left\langle\Delta_{1}^{\prime \prime}, \Delta_{2}^{\prime \prime}\right\rangle_{3}\right) f \Rightarrow\left(\kappa_{3}^{\lambda^{\prime}}\left\langle\Delta_{1}^{\prime \prime}, \Delta_{2}^{\prime \prime}\right\rangle_{2}\right)=f\left(\kappa_{3}^{\lambda \prime^{\prime}}\left\langle\Delta_{1}^{\prime \prime}, \Delta_{2}^{\prime \prime}\right\rangle_{3}\right)$.

Since, $\mathrm{f}$ is soft one-one, $\left(t_{\left(\mathfrak{a}^{\prime \prime}, \mathfrak{b}^{\prime \prime}\right)}^{e^{\prime \prime}}, \theta\right)_{2}=\kappa_{3}^{\lambda{ }^{\prime \prime}}\left\langle\Delta_{1}^{\prime \prime}, \Delta_{2}^{\prime \prime}\right\rangle_{3}$ implies that $\kappa_{3}^{\lambda \prime^{\prime}}\left\langle\Delta_{1}^{\prime \prime}, \Delta_{2}^{\prime \prime}\right\rangle_{2} \in f\left(\left\langle k_{1}, \theta\right\rangle\right), \kappa_{3}^{\lambda^{\prime}}\left\langle\Delta_{1}^{\prime \prime}, \Delta_{2}^{\prime \prime}\right\rangle_{2} \in f\left(\left\langle k_{2}, \theta\right\rangle\right)$ implies that $\kappa_{3}^{\lambda \prime^{\prime}}\left\langle\Delta_{1}^{\prime \prime}, \Delta_{2}^{\prime \prime}\right\rangle_{2} \in f\left(\left\langle k_{1}, \theta\right\rangle\right) \widetilde{\cap} f\left(\left\langle k_{2}, \theta\right\rangle\right)$. This is contradiction because $\left\langle k_{1}, \theta\right\rangle \widetilde{\cap}\left\langle k_{2}, \theta\right\rangle=0_{(\widetilde{\pi}, \theta)}$. Therefore, $f\left(\left\langle k_{1}, \theta\right\rangle\right) \widetilde{\cap} f\left(\left\langle k_{2}, \theta\right\rangle\right)=0_{(\widetilde{\pi}, \theta)}$. Finally, $\left.\kappa_{1}^{\lambda}\left\langle\Delta_{1}, \Delta_{2}\right\rangle_{1}\right\rangle \kappa_{1}^{\lambda}$ $\left\langle\Delta_{1}, \Delta_{2}\right\rangle_{2}$ or $\kappa_{1}^{\lambda}\left\langle\Delta_{1}, \Delta_{2}\right\rangle_{1} \prec \quad \kappa_{1}^{\lambda}\left\langle\Delta_{1}, \Delta_{2}\right\rangle_{2} \Rightarrow \kappa_{1}^{\lambda}\left\langle\Delta_{1}, \Delta_{2}\right\rangle_{1} \neq$ $\kappa_{1}^{\lambda}\left\langle\Delta_{1}, \Delta_{2}\right\rangle_{2}$ implies $\left.f\left(\kappa_{1}^{\lambda}\left\langle\Delta_{1}, \Delta_{2}\right\rangle_{1}\right)\right\rangle f\left(\kappa_{1}^{\lambda}\left\langle\Delta_{1}, \Delta_{2}\right\rangle_{2}\right)$ or $f$ $\left(\kappa_{1}^{\lambda}\left\langle\Delta_{1}, \Delta_{2}\right\rangle_{1}\right)<f\left(\kappa_{1}^{\lambda}\left\langle\Delta_{1}, \Delta_{2}\right\rangle_{2}\right) f \Rightarrow\left(\kappa_{1}^{\lambda}\left\langle\Delta_{1}, \quad \Delta_{2}\right\rangle_{1}\right) \neq f\left(\kappa_{1}^{\lambda}\right.$ $\left.\left\langle\Delta_{1}, \quad \Delta_{2}\right\rangle_{2}\right)$. Given a pair of points $\kappa_{2}^{\lambda^{\prime}}\left\langle\Delta_{1} /\right.$, $\left.\Delta_{2} /\right\rangle_{1}, \kappa_{2}^{\lambda^{\prime}}\left\langle\Delta_{1} /, \Delta_{2} /\right\rangle_{2} \in \tilde{Y}$, there exists $\kappa_{2}^{\lambda^{\prime}}\left\langle\Delta_{1} /, \Delta_{2} /\right\rangle_{1}$ $\neq \kappa_{2}^{\prime}\left\langle\Delta_{1} /, \Delta_{2} /\right\rangle_{2}$. We are able to find out mutually exclusive VS $*_{b}$-open sets $f\left(\left\langle k_{1}, \theta\right\rangle\right), f\left(\left\langle k_{2}, \theta\right\rangle\right) \in\left(\langle\widetilde{Y}\rangle, \mathfrak{F}_{1}, \mathfrak{F}_{2}, \theta\right)$ s.t., $\kappa_{2}^{\lambda^{\prime}}\left\langle\Delta_{1} /, \Delta_{2} /\right\rangle_{1} \in f\left(\left\langle k_{1}, \theta\right\rangle\right), \kappa_{2}^{\lambda^{\prime}}\left\langle\Delta_{1} /, \Delta_{2} /\right\rangle_{2} \in f\left(\left\langle k_{2}\right.\right.$, $\theta\rangle)$. This proves that $\left(\langle\widetilde{Y}\rangle, \mathfrak{F}_{1}, \mathfrak{F}_{2}, \theta\right)$ is $\operatorname{VSB} *_{b}$ Hausdorff space.

Theorem 10. Let $\left(\tilde{\pi}, \tau_{1}, \tau_{2}, \theta\right)$ be VSBTS and $\left(\langle\widetilde{Y}\rangle, \mathfrak{F}_{1}, \mathfrak{F}_{2}, \theta\right)$ be another VSBTS which satisfies one more condition of $V S B *_{b}$ Hausdorffness. Let $\langle\mathrm{f}, \theta\rangle$ : $\left(\widetilde{\pi}, \tau_{1}, \tau_{2}, \theta\right) \longrightarrow\left(\langle\widetilde{Y}\rangle, \mathfrak{\mho}_{1}, \mathfrak{\mho}_{2}, \theta\right)$ be a soft function, s.t., it is soft monotone and continuous. Then, $\left(\pi, \tau_{1}, \tau_{2}, \theta\right)$ is also characteristics of $V S B *_{b}$ Hausdorffness.

Proof. Suppose $\kappa_{1}^{\lambda}\left\langle\Delta_{1}, \Delta_{2}\right\rangle_{1}, \kappa_{1}^{\lambda}\left\langle\Delta_{1}, \Delta_{2}\right\rangle_{2} \in \tilde{\pi}$, such that either $\left.\kappa_{1}^{\lambda}\left\langle\Delta_{1}, \Delta_{2}\right\rangle_{1}\right\rangle \kappa_{1}^{\lambda}\left\langle\Delta_{1}, \Delta_{2}\right\rangle_{2}$ or $\kappa_{1}^{\lambda}\left\langle\Delta_{1}, \Delta_{2}\right\rangle_{1}\left\langle\kappa_{1}^{\lambda}\left\langle\Delta_{1}, \Delta_{2}\right\rangle_{2}\right.$. Let $\quad \kappa_{1}^{\lambda}\left\langle\Delta_{1}, \Delta_{2}\right\rangle_{1}>\kappa_{1}^{\lambda}\left\langle\Delta_{1}, \Delta_{2}\right\rangle_{2}$ or $\kappa_{1}^{\lambda}\left\langle\Delta_{1}, \Delta_{2}\right\rangle_{1}<\kappa_{1}^{\lambda}\left\langle\Delta_{1}, \Delta_{2}\right\rangle_{2}$ implies that $f\left(\kappa_{1}^{\lambda}\left\langle\Delta_{1}, \Delta_{2}\right\rangle_{1}\right)>f\left(\kappa_{1}^{\lambda}\left\langle\Delta_{1}, \Delta_{2}\right\rangle_{2}\right)$ or $f\left(\kappa_{1}^{\lambda}\right.$ $\left.\left\langle\Delta_{1}, \Delta_{2}\right\rangle_{1}\right)<f\left(\kappa_{1}^{\lambda}\left\langle\Delta_{1}, \Delta_{2}\right\rangle_{2}\right)$, respectively. Suppose $\kappa_{2}^{\lambda^{\prime}}\left\langle\Delta_{1} /\right.$, $\left.\Delta_{2} /\right\rangle_{1}, \kappa_{2}^{\prime}\left\langle\Delta_{1} /, \Delta_{2} /\right\rangle_{2} \in \tilde{Y}, \quad$ such that $\left.\kappa_{2}^{\lambda^{\prime}}\left\langle\Delta_{1} /, \quad \Delta_{2} /\right\rangle_{1}\right\rangle \kappa_{2}^{\lambda}$ $\left\langle\Delta_{1} /, \Delta_{2} /\right\rangle_{2}$ or $\kappa_{2}^{\lambda^{\prime}}\left\langle\Delta_{1} /, \Delta_{2} /\right\rangle_{1}\left\langle\kappa_{2}^{\lambda^{\prime}}\left\langle\Delta_{1} /, \Delta_{2} /\right\rangle_{2}\right.$. So, $\kappa_{2}^{\lambda^{\prime}}\left\langle\Delta_{1} /\right.$, $\left.\left.\Delta_{2} /\right\rangle_{1}\right\rangle \quad \kappa_{2}^{\lambda^{\prime}}\left\langle\Delta_{1} /, \Delta_{2} /\right\rangle_{2}$ or $\kappa_{2}^{\lambda^{\prime}}\left\langle\Delta_{1} /, \Delta_{2} /\right\rangle_{1}\left\langle\kappa_{2}^{\lambda^{\prime}}\left\langle\Delta_{1} /, \Delta_{2} /\right\rangle\right\rangle_{2}$, respectively, such that $\kappa_{2}^{\lambda^{\prime}}\left\langle\Delta_{1} /, \Delta_{2} /\right\rangle_{2}=f\left(\left(\begin{array}{ll}\kappa_{1} & \left\langle\Delta_{1}, \Delta_{2}\right\rangle\end{array}\right)\right.$ ), $\kappa_{2}^{\lambda^{\prime}}\left\langle\Delta_{1} /, \Delta_{2} /\right\rangle_{2}=f\left(\kappa_{1}^{\lambda}\left\langle\Delta_{1}, \Delta_{2}\right\rangle_{2}\right)$, such that $\kappa_{1}^{\lambda}\left\langle\Delta_{1}, \Delta_{2}\right\rangle_{1}$ $=f^{-1}\left(\kappa_{2}^{\lambda^{\prime}}\left\langle\Delta_{1} /, \Delta_{2} /\right\rangle_{1}\right)$ and $\kappa_{1}^{\lambda}\left\langle\Delta_{1}, \Delta_{2}\right\rangle_{2}=f^{-1}\left(\kappa_{2}^{\lambda^{\prime}}\left\langle\Delta_{1} /\right.\right.$, $\left.\left.\Delta_{2} /\right\rangle_{2}\right)$. Since $\kappa_{2}^{\lambda^{\prime}}\left\langle\Delta_{1} /, \Delta_{2} /\right\rangle_{1}, \kappa_{2}^{\lambda^{\prime}}\left\langle\Delta_{1} /, \quad \Delta_{2} /\right\rangle_{2} \in \tilde{Y}$, but $\left(\langle\widetilde{Y}\rangle, \mathfrak{F}_{1}, \mathfrak{F}_{2}, \theta\right)$ is VSB $*_{b}$ Hausdorff space. So according to definition, $\left.\kappa_{2}^{\lambda^{\prime}}\left\langle\Delta_{1} /, \Delta_{2} /\right\rangle_{1}\right\rangle \kappa_{2}^{\lambda^{\prime}}\left\langle\Delta_{1} /, \Delta_{2} /\right\rangle_{2}$ or $\kappa_{2}^{\lambda^{\prime}}\left\langle\Delta_{1} /, \Delta_{2} /\right\rangle_{1}\left\langle\kappa_{2}^{\lambda^{\prime}}\left\langle\Delta_{1} /, \Delta_{2} /\right\rangle_{2}\right.$. There definitely exists VS $*_{b}$-open sets $\left\langle k_{1}, \theta\right\rangle$ and $\left\langle k_{2}, \theta\right\rangle \in\left(\langle\widetilde{Y}\rangle, \mathfrak{F}_{1}, \mathfrak{F}_{2}, \theta\right)$, such that $\kappa_{2}^{\lambda^{\prime}}\left\langle\Delta_{1} /, \Delta_{2} /\right\rangle_{1} \in\left\langle k_{1}, \theta\right\rangle$ and $\kappa_{2}^{\lambda^{\prime}}\left\langle\Delta_{1} /, \Delta_{2} /\right\rangle_{2} \in\left\langle k_{2}, \theta\right\rangle$, and these two VS*b-open sets are disjoint. Since, $f^{-1}\left(\left\langle k_{1}, \theta\right\rangle\right)$ and $f^{-1}\left(\left\langle k_{2}, \theta\right\rangle\right)$ are $\mathrm{VS} *_{b}$ open in $\left(\tilde{\pi}, \tau_{1}, \tau_{2}, \theta\right)$. Now, $f^{-1}\left(\left\langle k_{1}, \theta\right\rangle\right) \widetilde{\cap} f^{-} \quad 1\left(\left\langle k_{1}, \theta\right\rangle\right)=f^{-1}$ $\left(\left\langle k_{1}, \theta\right\rangle \widetilde{\cap}\left\langle k_{2}, \theta\right\rangle\right)=f^{-1}(\tilde{\varnothing})=\underset{\tilde{\tilde{\pi}}, \partial)}{\widetilde{\sigma}} \quad$ and $\quad\left(k_{2}^{\lambda^{\prime}}\left\langle\Delta_{1} /\right.\right.$, $\left.\left.\Delta_{2} /\right\rangle\right)_{1} \in \quad\left\langle k_{1}, \theta\right\rangle \Rightarrow f^{-1}\left(\left(\kappa_{2}^{\lambda^{\prime}}\left\langle\Delta_{1} /, \quad \Delta_{2} /\right\rangle\right)_{1}\right) \in f^{-1}\left(\left\langle k_{1}\right.\right.$, $\theta\rangle) \Rightarrow \kappa_{1}^{\lambda}\left\langle\Delta_{1}, \Delta_{2}\right\rangle_{1} \in\left(\left\langle k_{1}, \theta\right\rangle\right), \quad \kappa_{2}^{\lambda^{\prime}}\left\langle\Delta_{1} /, \Delta_{2} /\right\rangle_{2} \in\left\langle k_{2}, \theta\right\rangle \Rightarrow$ $f^{-1}\left(\kappa_{2}^{\lambda^{\prime}}\left\langle\Delta_{1} /, \Delta_{2} /\right\rangle_{2}\right) \in f^{-1}\left(\left\langle k_{2}, \theta\right\rangle\right)$ imply that $\kappa_{1}^{\lambda}\left\langle\Delta_{1}\right.$, $\left.\Delta_{2}\right\rangle_{2} \in\left(\left\langle k_{2}, \theta\right\rangle\right) . \quad\left(\left\langle k_{1}, \theta\right\rangle\right), \quad \kappa_{1}^{\lambda}\left\langle\Delta_{1}, \Delta_{2}\right\rangle_{2} \in f^{-1}\left(\left\langle k_{2}, \theta\right\rangle\right)$. Accordingly, VSBTS is $*_{b}$ Hausdorff space.

Theorem 11. Let $\left(\tilde{\pi}, \tau_{1}, \tau_{2}, \theta\right)$ be VSBTS and $\left(\langle\widetilde{Y}\rangle, \mathfrak{F}_{1}, \mathfrak{F}_{2}, \theta\right)$ be another VSBTS. Let $\langle f, \theta\rangle$ : $\left(\widetilde{\pi}, \tau_{1}, \tau_{2}, \theta\right) \longrightarrow\left(\langle\widetilde{Y}\rangle, \mathfrak{F}_{1}, \mathfrak{F}_{2}, \theta\right)$ be a soft mapping. Let $\left(\langle\widetilde{Y}\rangle, \mathfrak{F}_{1}, \mathfrak{F}_{2}, \theta\right)$ be VSB* $*_{b}$ Hausdorff space; then, it is guaranteed that $\left\{\left(\left(\kappa_{1}^{\lambda}\left\langle\Delta_{1}, \Delta_{2}\right\rangle\right), \quad \kappa_{2}^{\lambda^{\prime}}\left\langle\Delta_{1} /, \Delta_{2} /\right\rangle\right): f\right.$ $\left.\left(\kappa_{1}^{\lambda}\left\langle\Delta_{1}, \Delta_{2}\right\rangle\right)=f\left(\kappa_{2}^{\lambda^{\prime}}\left\langle\Delta_{1} /, \Delta_{2} /\right\rangle\right)\right\}$ is a VS $*_{b}$-closed subset of $\left(\tilde{\pi}, \tau_{1}, \tau_{2}, \theta\right) \times\left(\langle\widetilde{Y}\rangle, \widetilde{\mho}_{1}, \mathfrak{\mho}_{2}, \theta\right)$.

Proof. Given that $\left(\tilde{\pi}, \tau_{1}, \tau_{2}, \theta\right)$ be VSBTS and $\left(\langle\widetilde{Y}\rangle, \mathfrak{F}_{1}, \mathfrak{F}_{2}, \theta\right)$ be another VSBTS, let $\langle f, \theta\rangle:\left(\widetilde{\pi}, \tau_{1}\right.$, $\left.\tau_{2}, \theta\right) \times\left(\langle\widetilde{Y}\rangle, \mathfrak{F}_{1}, \mathfrak{\mho}_{2}, \theta\right)$ be a soft mapping, such that it is continuous mapping. $\left(\langle\widetilde{Y}\rangle, \mathfrak{F}_{1}, \mathfrak{F}_{2}, \theta\right)$ is VSB $*_{b}$ Hausdorff space. Then, we will prove that $\left\{\left(\left(\left(\kappa_{1}^{\lambda}\left\langle\Delta_{1}, \Delta_{2}\right\rangle\right)\right), \kappa_{2}^{\lambda^{\prime}}\left\langle\Delta_{1} /\right.\right.\right.$, $\left.\left.\left.\Delta_{2} /\right\rangle\right): f\left(\left(\kappa_{1}^{\lambda}\left\langle\Delta_{1}, \Delta_{2}\right\rangle\right)\right)=f\left(\kappa_{2}^{\lambda^{\prime}}\left\langle\Delta_{1} /, \Delta_{2} /\right\rangle\right)\right\}$ is a VS $*_{b}$-closed subset of $\left(\tilde{\pi}, \tau_{1}, \tau_{2}, \theta\right) \times\left(\langle\tilde{Y}\rangle, \mathfrak{\mho}_{1}, \mathfrak{\mho}_{2}, \theta\right)$. Equivalently, we will prove that $\left\{\left(\kappa_{1}^{\lambda}\left\langle\Delta_{1}, \Delta_{2}\right\rangle\right.\right.$, $\left.\left.\kappa_{2}^{\lambda^{\prime}}\left\langle\Delta_{1} /, \Delta_{2} /\right\rangle\right): f\left(\left(\kappa_{1}^{\lambda}\left\langle\Delta_{1}, \Delta_{2}\right\rangle\right)\right)=\left(\kappa_{2}^{\lambda^{\prime}}\left\langle\Delta_{1} /, \Delta_{2} /\right\rangle\right)\right\}^{c}$ is a $V S *_{b}$-open subset of $\left(\widetilde{\pi}, \tau_{1}, \tau_{2}, \theta\right) f\left(\langle\tilde{Y}\rangle, \mathfrak{F}_{1}, \mathfrak{F}_{2}, \theta\right)$. Let $\left(\left(\kappa_{1}^{\lambda}\left\langle\Delta_{1}, \Delta_{2}\right\rangle\right), \kappa_{2}^{\lambda^{\prime}}\left\langle\Delta_{1} /, \quad \Delta_{2} /\right\rangle\right) \in\left\{\left(\kappa_{1}^{\lambda}\left\langle\Delta_{1}, \Delta_{2}\right\rangle\right), \quad\left(\kappa_{2}^{\lambda^{\prime}}\left\langle\Delta_{1} /\right.\right.\right.$, $\left.\left.\Delta_{2} /\right\rangle\right)$ with $\left.\quad\left(\kappa_{1}^{\lambda}\left\langle\Delta_{1}, \Delta_{2}\right\rangle\right)\right\rangle f\left(\kappa_{2}^{\lambda^{\prime}}\left\langle\Delta_{1} / \quad, \Delta_{2} /\right\rangle\right): f\left(\kappa_{1}^{\lambda}\left\langle\Delta_{1}\right.\right.$, $\left.\left.\left.\left.\Delta_{2}\right\rangle\right)\right\rangle f\left(\kappa_{2}^{\lambda^{\prime}}\left\langle\Delta_{1} /, \Delta_{2} /\right\rangle\right)\right\}^{c}$ or $\left(\left(\kappa_{1}^{\lambda}\left\langle\Delta_{1}, \quad \Delta_{2}\right\rangle\right), \kappa_{2}^{\lambda^{\prime}}\left\langle\Delta_{1} /, \Delta_{2} /\right\rangle\right) \epsilon$ $\left\{\left(\kappa_{1}^{\lambda}\left\langle\Delta_{1}, \Delta_{2}\right\rangle\right),\left(\kappa_{2}^{\lambda^{\prime}}\left\langle\Delta_{1} /, \Delta_{2} /\right\rangle\right)\right.$ with $\quad\left(\kappa_{1}^{\lambda}\left\langle\Delta_{1}, \Delta_{2}\right\rangle\right)<f\left(\kappa_{2}^{\lambda^{\prime}}\right.$ $\left.\left.\left\langle\Delta_{1} /, \Delta_{2} /\right\rangle\right): f\left(\kappa_{1}^{\lambda}\left\langle\Delta_{1}, \Delta_{2}\right\rangle\right)<f\left(\kappa_{2}^{\lambda^{\prime}}\left\langle\Delta_{1} /, \Delta_{2} /\right\rangle\right)\right\}^{c}$. Then, $f\left(\kappa_{1}^{\lambda}\right.$ $\left.\left.\left\langle\Delta_{1}, \Delta_{2}\right\rangle\right)\right\rangle f\left(\kappa_{2}^{\prime}\left\langle\Delta_{1} /, \Delta_{2} /\right\rangle\right) \quad$ or $\quad\left\langle f\left(\kappa_{1}^{\lambda}\left\langle\Delta_{1}, \Delta_{2}\right\rangle\right)<f\right.$ $\left(\kappa_{2}^{\lambda^{\prime}}\left\langle\Delta_{1} /, \Delta_{2} /\right\rangle\right)$ accordingly. Since, $\left(\langle\widetilde{Y}\rangle, \mathfrak{F}_{1}, \mathfrak{F}_{2}, \theta\right)$ is $*_{b}$ V-Hausdorff space. Certainly, $f\left(\kappa_{1}^{\lambda}\left\langle\Delta_{1}, \Delta_{2}\right\rangle\right), f$ $\left(\kappa_{2}^{\lambda^{\prime}}\left\langle\Delta_{1} /, \Delta_{2} /\right\rangle\right)$ are points of $\left(\langle\widetilde{Y}\rangle, \mathfrak{F}_{1}, \mathfrak{F}_{2}, \theta\right)$, and there exists VS $*_{b}$-open sets $\langle\mathscr{G}, \theta\rangle,\langle k, \theta\rangle \in\left(\langle\widetilde{Y}\rangle, \mathfrak{F}_{1}, \mathfrak{F}_{2}, \theta\right)$, such that $f\left(\left(\kappa_{1}^{\lambda}\left\langle\Delta_{1}, \Delta_{2}\right\rangle\right)\right) \in\langle\mathscr{G}, \theta\rangle, f\left(\kappa_{1}^{\lambda}\left\langle\Delta_{1}, \Delta_{2}\right\rangle\right) \in\langle k, \theta\rangle$, provided $\langle\mathscr{G}, \theta\rangle \widetilde{\cap}\langle k, \theta\rangle=\widetilde{0 \widetilde{\tilde{\pi}, \theta)^{Y}}}$. Since $\langle f, \theta\rangle$ is soft continuous, $f^{-1}(\langle\mathscr{G}, \theta\rangle)$ and $f^{-1}(\langle k, \theta\rangle)$ are VS $*_{b}$-open sets in $\left\langle\tilde{\pi}, \tau_{1}, \tau_{2}, \theta\right\rangle$, supposing $\kappa_{1}^{\lambda}\left\langle\Delta_{1}, \Delta_{2}\right\rangle$ and $\kappa_{2}^{\lambda^{\prime}}\left\langle\Delta_{1} /, \Delta_{2} /\right\rangle$, respectively, and so, $f^{-1}(\langle\mathscr{G}, \theta\rangle) \times f^{-1}(\langle\mathscr{H}, \theta\rangle)$ is a basic VS $*_{b}$-open set in $\left(\widetilde{\pi}, \tau_{1}, \tau_{2}, \theta\right) \times\left(\langle\widetilde{Y}\rangle, \mathfrak{F}_{1}, \mathfrak{F}_{2}, \theta\right)$ containing $\left(\left(\kappa_{1}^{\lambda}\left\langle\Delta_{1}, \Delta_{2}\right\rangle\right), \kappa_{2}^{\lambda^{\prime}}\left\langle\Delta_{1} /, \Delta_{2} /\right\rangle\right)$. Since $\langle\mathscr{G}, \theta\rangle \widetilde{\cap}\langle k, \theta\rangle=\widetilde{0_{\theta_{Y}}}$, it is clear by the definition of $\left\{\left(\left(\kappa_{1}^{\lambda}\left\langle\Delta_{1}, \Delta_{2}\right\rangle\right)\right.\right.$, $\left.\left.\kappa_{2}^{\lambda^{\prime}}\left\langle\Delta_{1} /, \Delta_{2} /\right\rangle\right): f\left(\kappa_{1}^{\lambda}\left\langle\Delta_{1}, \Delta_{2}\right\rangle\right)=f\left(\kappa_{2}^{\lambda^{\prime}}\left\langle\Delta_{1} /, \Delta_{2} /\right\rangle\right)\right\}$, that is, $\left\{f^{-1}\langle\mathscr{G}, \theta\rangle\right.$ and $f^{-1}(\langle k, \quad \theta\rangle\} \widetilde{\cap}\left\{\left(\left(\kappa_{1}^{\lambda}\left\langle\Delta_{1}, \Delta_{2}\right\rangle\right), \quad \kappa_{2}^{\lambda^{\prime}}\left\langle\Delta_{1} /\right.\right.\right.$, $\left.\left.\left.\Delta_{2} /\right\rangle\right): f(\kappa)=f\left(\kappa_{2}^{\lambda^{\prime}}\left\langle\Delta_{1} /, \Delta_{2} /\right\rangle\right)\right\}=0_{(\pi, \theta)}, \quad$ that is, $f^{-1}$ $(\langle\mathscr{G}, \theta\rangle) \times f^{-1}(\langle k, \theta\rangle) \Subset\left\{\left(\kappa_{1}^{\lambda}\left\langle\Delta_{1}, \quad \Delta_{2}\right\rangle, \kappa_{2}^{\lambda^{\prime}}\left\langle\Delta_{1} /, \Delta_{2} /\right\rangle\right):\right.$ $\left.f\left(\kappa_{1}^{\lambda}\left\langle\Delta_{1}, \Delta_{2}\right\rangle\right)=f\left(\kappa_{2}^{\prime}\left\langle\Delta_{1} /, \Delta_{2} /\right\rangle\right)\right\}^{c}$. Hence, $\left\{\left(\kappa_{1}^{\lambda}\left\langle\Delta_{1}\right.\right.\right.$, $\left.\left.\left.\Delta_{2}\right\rangle, \kappa_{2}^{\lambda^{\prime}}\left\langle\Delta_{1} /, \quad \Delta_{2} /\right\rangle\right): f\left(\kappa_{1}^{\lambda}\left\langle\Delta_{1}, \Delta_{2}\right\rangle\right)=f\left(\kappa_{2}^{\lambda^{\prime}}\left\langle\Delta_{1} /, \Delta_{2} /\right\rangle\right)\right\}^{c}$ implies that $\left\{\left(\kappa_{1}^{\lambda}\left\langle\Delta_{1}, \Delta_{2}\right\rangle, \kappa_{2}^{\lambda^{\prime}}\left\langle\Delta_{1} /\right.\right.\right.$, 
$\left.\left.\left.\Delta_{2} /\right\rangle\right): f\left(\kappa_{1}^{\lambda}\left\langle\Delta_{1}, \Delta_{2}\right\rangle\right)=f\left(\kappa_{2}^{\lambda}\left\langle\Delta_{1} /, \Delta_{2} /\right\rangle\right)\right\} \quad$ is $\quad \mathrm{VS} *_{b}$ closed.

Theorem 12. Let $\left(\tilde{\pi}, \tau_{1}, \tau_{2}, \theta\right)$ be VSBTS and $\left(\langle\widetilde{Y}\rangle, \mathfrak{F}_{1}, \mathfrak{F}_{2}, \theta\right)$ be another VSBTS. Let $\langle f, \theta\rangle:\left(\tilde{\pi}, \tau_{1}, \tau_{2}, \theta\right) \times\left(\langle\widetilde{Y}\rangle, \mathfrak{F}_{1}, \mathfrak{F}_{2}, \theta\right)$ be VS* $*_{b}$ open mapping, such that it is onto. If the soft set $\left\{\left(\kappa_{1}^{\lambda}\left\langle\Delta_{1}, \Delta_{2}\right\rangle\right.\right.$, $\left.\left.\kappa_{2}^{\lambda^{\prime}}\left\langle\Delta_{1} /, \Delta_{2} /\right\rangle\right): f\left(\left(\kappa_{1}^{\lambda}\left\langle\Delta_{1}, \Delta_{2}\right\rangle\right)\right)=f\left(\left(\kappa_{2}^{\lambda^{\prime}}\left\langle\Delta_{1} /, \Delta_{2} /\right\rangle\right)\right)\right\}$ is $N S *_{b}$ closed in $\left(\tilde{\pi}, \tau_{1}, \tau_{2}, \theta\right) \times\left(\langle\tilde{Y}\rangle, \mathfrak{\mho}_{1}, \mathfrak{\mho}_{2}, \theta\right)$, then $\left\langle\tilde{\pi}, \tau_{1}, \tau_{2}, \theta\right\rangle$ will behave as VSB* $*_{b}$ Hausdorff space.

Proof. Suppose $f\left(\kappa_{1}^{\lambda}\left\langle\Delta_{1}, \Delta_{2}\right\rangle\right), f\left(\kappa_{2}^{\lambda^{\prime}}\left\langle\Delta_{1} /, \Delta_{2} /\right\rangle\right)$ be two points of $\tilde{Y}$, such that either $f\left(\kappa_{1}^{\lambda}\left\langle\Delta_{1}, \Delta_{2}\right\rangle\right)>f$ $\left(\kappa_{2}^{\lambda^{\prime}}\left\langle\Delta_{1} /, \Delta_{2} /\right\rangle\right)$ or $\left\langle f\left(\kappa_{1}^{\lambda}\left\langle\Delta_{1}, \Delta_{2}\right\rangle\right)<f\left(\kappa_{2}^{\lambda^{\prime}}\left\langle\Delta_{1} /, \Delta_{2} /\right\rangle\right)\right.$. Then, $\left(\kappa_{1}^{\lambda}\left\langle\Delta_{1}, \Delta_{2}\right\rangle, \quad \kappa_{2}^{\lambda^{\prime}}\left\langle\Delta_{1} /, \Delta_{2} /\right\rangle\right) \notin\left\{\left(\kappa_{2}^{\lambda^{\prime}}\left\langle\Delta \quad{ }_{1} /, \Delta_{2} /\right\rangle\right)\right.$ with $\kappa_{1}^{\lambda}$ $\left.\left.\left.\left\langle\Delta_{1}, \Delta_{2}\right\rangle\right\rangle\left(\kappa_{2}^{\lambda^{\prime}}\left\langle\Delta_{1} /, \Delta_{2} /\right\rangle\right): f\left(\kappa_{1}^{\lambda}\left\langle\Delta_{1}, \Delta_{2}\right\rangle\right)\right\rangle f\left(\kappa_{2}^{\lambda^{\prime}}\left\langle\Delta_{1} /, \Delta_{2} /\right\rangle\right)\right\}$ or $\left(\kappa_{1}^{\lambda}\left\langle\Delta_{1}, \Delta_{2}\right\rangle, \kappa_{2}^{\lambda^{\prime}}\left\langle\Delta_{1} /, \Delta_{2} /\right\rangle\right) \notin\left\{\left(\kappa_{1}^{\lambda}\left\langle\Delta_{1}, \Delta_{2}\right\rangle\right)\right.$ with $\kappa_{1}^{\lambda}\left\langle\Delta_{1}\right.$, $\left.\left.\Delta_{2}\right\rangle<\left(\kappa_{2}^{\lambda^{\prime}}\left\langle\Delta_{1} /, \Delta_{2} /\right\rangle\right): f\left(\kappa_{1}^{\lambda}\left\langle\Delta_{1}, \Delta_{2}\right\rangle\right)<f\left(\kappa_{2}^{\lambda^{\prime}}\left\langle\Delta_{1} /, \Delta_{2} /\right\rangle\right)\right\}$, that is, $\quad\left(\kappa_{1}^{\lambda}\left\langle\Delta_{1}, \Delta_{2}\right\rangle, \kappa_{2}^{\lambda^{\prime}}\left\langle\Delta_{1} /, \Delta_{2} /\right\rangle\right) \notin\left\{\left(\kappa_{1}^{\lambda}\left\langle\Delta_{1}, \Delta_{2}\right\rangle, \quad \kappa_{2}^{\lambda^{\prime}}\left\langle\Delta_{1} /\right.\right.\right.$, $\left.\left.\Delta_{2} /\right\rangle\right)$ with $\left.\left.\kappa_{1}^{\lambda}\left\langle\Delta_{1}, \Delta_{2}\right\rangle\right\rangle\left(\kappa_{2}^{\lambda^{\prime}}\left\langle\Delta_{1} /, \Delta_{2}\right\rangle\right): f\left(\kappa_{1}^{\lambda}\left\langle\Delta_{1}, \Delta_{2}\right\rangle\right)\right\rangle f\left(\kappa_{2}^{\lambda}\right.$ $\left.\left.\left\langle\Delta_{1} / \Delta_{2} /\right\rangle\right)\right\} \quad$ or $\quad\left(\kappa_{1}^{\lambda}\left\langle\Delta_{1}, \Delta_{2}\right\rangle, \kappa_{2}^{\lambda^{\prime}}\left\langle\Delta_{1} /, \Delta_{2} /\right\rangle\right) \notin\left\{\left(\kappa_{1}^{\lambda}\left\langle\Delta_{1}, \Delta_{2}\right\rangle\right.\right.$, $\left.\kappa_{2}^{\lambda^{\prime}}\left\langle\Delta_{1} /, \Delta_{2} /\right\rangle\right)$ with $\quad \kappa_{1}^{\lambda}\left\langle\Delta_{1}, \Delta_{2}\right\rangle<\left(\kappa_{2}^{\lambda^{\prime}} \quad\left\langle\Delta_{1} / \Delta_{2} /\right\rangle\right): f\left(\kappa_{1}^{\lambda}\left\langle\Delta_{1}\right.\right.$, $\left.\left.\left.\Delta_{2}\right\rangle\right)<f\left(\kappa_{2}^{\lambda^{\prime}}\left\langle\Delta_{1} /, \Delta_{2} /\right\rangle\right)\right\}^{c}$. Since, $\left(\kappa_{1}^{\lambda}\left\langle\Delta_{1}, \Delta_{2}\right\rangle, \kappa_{2}^{\lambda^{\prime}}\left\langle\Delta_{1} /, \Delta_{2} /\right\rangle\right) \epsilon$ $\left\{\left(\kappa_{1}^{\lambda}\left\langle\Delta_{1}, \quad \Delta_{2}\right\rangle, \kappa_{2}^{\lambda^{\prime}}\left\langle\Delta_{1} /, \Delta_{2} /\right\rangle\right)\right.$ with $\left.\quad \kappa_{1}^{\lambda}\left\langle\Delta_{1}, \Delta_{2}\right\rangle\right\rangle\left(\kappa_{2}^{\lambda}\right.$ $\left.\left.\left.\left\langle\Delta_{1} /, \Delta_{2} /\right\rangle\right): f\left(\kappa_{1}^{\lambda}\left\langle\Delta_{1}, \Delta_{2}\right\rangle\right)\right\rangle f\left(\kappa_{2}^{\lambda^{\prime}}\left\langle\Delta_{1} /, \Delta_{2} /\right\rangle\right)\right\}^{c}$ or $\left(\kappa_{1}^{\lambda}\left\langle\Delta_{1}\right.\right.$, $\left.\left.\Delta_{2}\right\rangle, \kappa_{2}^{\lambda^{\prime}}\left\langle\Delta \quad{ }_{1} /, \Delta_{2} /\right\rangle\right) \in\left\{\left(\kappa_{1}^{\lambda}\left\langle\Delta_{1}, \Delta_{2}\right\rangle, \quad \kappa_{2}^{\lambda^{\prime}}\left\langle\Delta_{1} /, \Delta_{2} /\right\rangle\right)\right.$ with $\kappa_{1}^{\lambda}\left\langle\Delta_{1}, \Delta_{2}\right\rangle<\left(\kappa_{2}^{\lambda^{\prime}} \quad\left\langle\Delta_{1} /, \Delta_{2} /\right\rangle\right): f\left(\kappa_{1}^{\lambda}\left\langle\Delta_{1}, \Delta_{2}\right\rangle\right)<f\left(\kappa_{2}^{\lambda^{\prime}}\left\langle\Delta_{1} /\right.\right.$, $\left.\left.\left.\Delta_{2} /\right\rangle\right)\right\}^{c}$ is soft in $\left\langle\tilde{\pi}, \tau_{1}, \tau_{2}, \theta\right\rangle \times\left\langle\langle\widetilde{Y}\rangle, \mathfrak{F}_{1}, \mathfrak{F}_{2}, \theta\right\rangle$, then there exists VS* $*_{b}$-open sets $\langle\mathscr{G}, \theta\rangle$ and $\langle\mathscr{G}, \theta\rangle$ in $\left\langle\tilde{\pi}, \tau_{1}, \tau_{2}, \theta\right\rangle$, such that $\left(\kappa_{1}^{\lambda}\left\langle\Delta_{1}, \Delta_{2}\right\rangle, \kappa_{2}^{\lambda^{\prime}}\left\langle\Delta_{1} /, \Delta_{2} /\right\rangle\right) \in\langle\mathscr{G}, \quad \theta\rangle \times\langle k$, $\theta\rangle \Subset\left\{\left(\left(\kappa_{1}^{\lambda}\left\langle\Delta_{1}, \Delta_{2}\right\rangle\right), \quad \kappa_{2}^{\lambda^{\prime}}\left\langle\Delta_{1} /, \Delta_{2} /\right\rangle\right)\right.$ with $\quad \kappa_{1}^{\lambda}\left\langle\Delta_{1}, \Delta_{2}\right\rangle>\kappa_{2}^{\lambda^{\prime}}$ $\left.\left.\left.\left\langle\Delta_{1} /, \Delta_{2}\right\rangle\right\rangle: \quad f\left(\left(\kappa_{1}^{\lambda}\left\langle\Delta_{1}, \Delta_{2}\right\rangle\right)\right)\right\rangle f\left(\kappa_{2}^{\lambda^{\prime}}\left\langle\Delta_{1} /, \Delta_{2} /\right\rangle\right)\right\}^{c} \quad$ or $\left(\kappa_{1}^{\lambda}\left\langle\Delta_{1}, \Delta_{2}\right\rangle, \quad \kappa_{2}^{\lambda^{\prime}}\left\langle\Delta_{1} /, \Delta_{2} /\right\rangle\right) \in\langle\mathscr{G}, \theta\rangle \times\langle k, \theta\rangle \Subset\left\{\left(\left(\kappa_{1}^{\lambda}\left\langle\Delta_{1}\right.\right.\right.\right.$, $\left.\left.\left.\Delta_{2}\right\rangle\right), \kappa_{2}^{\lambda^{\prime}}\left\langle\Delta_{1} /, \Delta_{2} /\right\rangle\right)$ with $\quad \kappa_{1}^{\lambda}\left\langle\Delta_{1}, \Delta_{2}\right\rangle\left\langle\kappa_{2}^{\lambda^{\prime}} \quad\left\langle\Delta_{1} /, \Delta_{2}\right\rangle\right\rangle: f$ $\left.\left(\left(\kappa_{1}^{\lambda}\left\langle\Delta_{1}, \Delta_{2}\right\rangle\right)\right)<f\left(\kappa_{2}^{\prime}\left\langle\Delta_{1} /, \Delta_{2} /\right\rangle\right)\right\}^{c}$. Then, since $\mathrm{f}$ is $\mathrm{VS} *_{b}$ open, $f(\langle\mathscr{G}, \theta\rangle))$ and $f(\langle k, \theta\rangle)$ are $\mathrm{VS} *_{b}$-open sets in $\left(\langle\widetilde{Y}\rangle, \mathfrak{F}_{1}, \mathfrak{\mho}_{2}, \theta\right) \quad$ containing $f\left(\kappa_{1}^{\lambda}\left\langle\Delta_{1}, \Delta_{2}\right\rangle\right)$ and $f\left(\kappa_{2}^{\lambda^{\prime}}\left\langle\Delta_{1} /, \Delta_{2} /\right\rangle\right), \quad$ respectively, and $f(\langle\mathscr{G}, \theta\rangle) \widetilde{\cap} f$ $(\langle k, \theta\rangle)=\widetilde{0_{(\widetilde{\pi}, \theta)}} ; \quad$ otherwise, $\quad f(\langle\mathscr{G}, \theta\rangle) \times f(\langle k, \theta\rangle) \widetilde{\cap}$ $\left\{\left(\left(\kappa_{1}^{\lambda}\left\langle\Delta_{1}, \Delta_{2}\right\rangle\right)\right)\right.$ ) with $\left.\left.\left(\kappa_{1}^{\lambda}\left\langle\Delta_{1}, \Delta_{2}\right\rangle\right)\right\rangle \kappa_{2}^{\lambda^{\prime}}\left\langle\Delta_{1} /, \Delta_{2}\right\rangle\right\rangle: f\left(\left(\kappa_{1}^{\lambda}\left\langle\Delta_{1}\right.\right.\right.$, $\left.\left.\left.\left.\Delta_{2}\right\rangle\right)\right)>f\left(\kappa_{2}^{\lambda^{\prime}}\left\langle\Delta_{1} /, \Delta_{2} /\right\rangle\right)\right\} \quad$ or $\quad\left(\left(\left(\kappa_{1}^{\lambda}\left\langle\Delta_{1}, \Delta_{2}, \Delta_{3}\right\rangle\right), \quad \kappa_{2}^{\lambda^{\prime}}\right.\right.$ $\left.\left.\left\langle\Delta_{1} /, \Delta_{2}\right\rangle\right)\right) \notin\left\{\left(\left(\kappa_{1}^{\lambda} \quad\left\langle\Delta_{1}, \Delta_{2}\right\rangle\right), \kappa_{2}^{\lambda^{\prime}}\left\langle\Delta_{1} /, \Delta_{2} /\right\rangle\right)\right.$ with $\quad\left(\kappa_{1}^{\lambda}\left\langle\Delta_{1}\right.\right.$, $\left.\left.\Delta_{2}\right\rangle\right)\left\langle\kappa_{2}^{\lambda^{\prime}} \quad\left\langle\Delta_{1} / \Delta_{2} /\right\rangle: f\left(\left(\kappa_{1}^{\lambda}\left\langle\Delta_{1}, \Delta_{2}\right\rangle\right)\right)<f\left(\kappa_{2}^{\lambda^{\prime}}\left\langle\Delta_{1} /, \Delta_{2} /\right\rangle\right)\right\}=$ $\widetilde{0_{(\tilde{\pi}, \theta)}}$. It follows that $\left\langle\langle\widetilde{Y}\rangle, \mathfrak{F}_{1}, \mathfrak{\mho}_{2}, \theta\right\rangle$ is $\operatorname{VSB} *_{b}$ Hausdorff space.

Theorem 13. Let $\left\langle\tilde{\pi}, \tau_{1}, \tau_{2}, \theta\right\rangle$ be VSB second countable space, and let $\langle f, \theta\rangle$ be a VS uncountable subset of $\left\langle\widetilde{\pi}, \tau_{1}, \tau_{2}, \theta\right\rangle$. Then, at least one point of $\langle f, \theta\rangle$ is a soft limit point of $\langle f, \theta\rangle$.

Proof. Let $\mathfrak{W}=\mathscr{B}_{1}, \mathscr{B}_{2}, \mathscr{B}_{3}, \mathscr{B}_{4}, \ldots, \mathscr{B}_{n}: n \in \mathbb{N}$ for $\left\langle\tilde{\pi}, \tau_{1}\right.$, $\left.\tau_{2}, \theta\right\rangle$.

Let, if possible, no point of $\langle f, \theta\rangle$ be a soft limit point of $\langle f, \theta\rangle$. Then, for each $\kappa_{1}^{\lambda}\left\langle\Delta_{1}, \Delta_{2}\right\rangle \in\langle f, \theta\rangle$, there exists a VS $*_{b}$-open set $\langle\rho, \theta\rangle_{\left(\kappa_{1}^{\curlywedge}\left\langle\Delta_{1}, \Delta_{2}\right\rangle\right)}$, such that $\kappa_{1}^{\lambda}\left\langle\Delta_{1}, \Delta_{2}\right\rangle \epsilon$ $\langle\rho, \theta\rangle_{\left(\kappa_{1}^{\lambda}\left\langle\Delta_{1}, \Delta_{2}\right\rangle\right)}$ and $\langle\rho, \theta\rangle_{\left(\kappa_{1}^{\lambda}\left\langle\Delta_{1}, \Delta_{2}\right\rangle\right)} \widetilde{\cap}\langle f, \theta\rangle=\left\{\left(\kappa_{1}^{\lambda}\left\langle\Delta_{1}\right.\right.\right.$, $\left.\left.\left.\Delta_{2}\right\rangle\right)\right\}$. Since $\mathfrak{W}$ is soft base $\exists \mathscr{B}_{\left.n_{\left(\alpha_{1}\right\rangle}\left\langle\Delta_{1}, \Delta_{2}\right\rangle\right)} \in \mathfrak{W}$, such that $\left(\kappa_{1}^{\lambda}\left\langle\Delta_{1}, \Delta_{2}\right\rangle\right) \in \mathscr{B}_{n\left(\kappa_{1}^{\lambda}\left\langle\Delta_{1}, \Delta_{2}\right\rangle\right)} \Subset\langle\rho, \theta\rangle_{\left(\kappa_{1}^{\lambda}\left\langle\Delta_{1}, \Delta_{2}\right\rangle\right)}$. Therefore, $\mathscr{B}$ $n_{\left(\hat{\alpha}_{1}^{>}\left\langle\Delta_{1}, \Delta_{2}\right\rangle\right)} \widetilde{n}\langle f, \theta\rangle \Subset \rho, \theta_{\left(\kappa_{1}^{\lambda}\left\langle\Delta_{1}, \Delta_{2}\right\rangle\right)} \widetilde{\cap}\langle f, \theta\rangle=\left\{\left(\kappa_{1}^{\lambda}\left\langle\Delta_{1}, \Delta_{2}\right\rangle\right)\right\}$.

Moreover, if $\kappa_{1}^{\lambda}\left\langle\Delta_{1}, \Delta_{2}\right\rangle_{1}$ and $\kappa_{1}^{\lambda}\left\langle\Delta_{1}, \Delta_{2}\right\rangle_{2}$ be any two VS points, such that $\kappa_{1}^{\lambda}\left\langle\Delta_{1}, \Delta_{2}\right\rangle_{1} \neq \kappa_{1}^{\lambda}\left\langle\Delta_{1}, \Delta_{2}\right\rangle_{2}$ which means either $\left.\kappa_{1}^{\lambda}\left\langle\Delta_{1}, \Delta_{2}\right\rangle_{1}\right\rangle \kappa_{1}^{\lambda}\left\langle\Delta_{1}, \Delta_{2}\right\rangle_{2}$ or $\kappa_{1}^{\lambda}\left\langle\Delta_{1}, \Delta_{2}\right\rangle_{1}\left\langle\kappa_{1}^{\lambda}\left\langle\Delta_{1}, \Delta_{2}\right\rangle_{2}\right.$, then there exist $\mathscr{B}_{n_{\left(k_{1}^{\lambda}\left\langle\Delta_{1}, \Delta_{2}\right\rangle\right)_{1}}}$ and $\mathscr{B}_{\left.n_{\left(k_{1}\right\rangle}\left\langle\Delta_{1}, \Delta_{2}\right\rangle\right)_{2}}$ in $\mathfrak{W}$, such that $\mathscr{B}_{n_{\left(\kappa_{1}^{\lambda}\left\langle\Delta_{1}, \Delta_{2}\right\rangle\right\rangle_{1}}} \widetilde{\cap}\langle f, \theta\rangle=\left\{\left(\kappa_{1}^{\lambda}\left\langle\Delta_{1}, \Delta_{2}\right\rangle\right)_{1}\right\} \quad$ and $\mathscr{B}_{n_{\left(\hat{\gamma}_{1}\right\rangle}\left\langle\Delta_{1}, \Delta_{2}\right\rangle_{2}}$ $\widetilde{n}\langle f, \theta\rangle=\left\{\left(\kappa_{1}^{\lambda}\left\langle\Delta_{1}, \Delta_{2}\right\rangle\right)_{2}\right\} . \quad$ Now, $\quad\left(\kappa_{1}^{\lambda}\left\langle\Delta_{1}, \Delta_{2}\right\rangle\right)_{1} \neq\left(\kappa_{1}^{\lambda}\right.$ $\left.\left\langle\Delta_{1}, \Delta_{2}\right\rangle\right)_{2}$, which guarantees that $\left\{\left(\kappa_{1}^{\lambda}\left\langle\Delta_{1}, \Delta_{2}\right\rangle\right)_{1}\right\} \neq\left\{\left(\kappa_{1}^{\lambda}\left\langle\Delta_{1}, \Delta_{2}\right\rangle\right)_{2}\right\}$, which implies that $\mathscr{B}_{\left.n_{\left(\kappa_{1}\right\rangle}\left\langle\Delta_{1}, \Delta_{2}\right\rangle\right)_{1}} \widetilde{\cap}\langle f, \theta\rangle \neq \mathscr{B}_{n_{\left(\lambda_{1}^{\lambda}\left\langle\Delta_{1}, \Delta_{2}\right\rangle\right)_{2}}} \widetilde{\cap}\langle f, \theta\rangle$, which implies $\mathscr{B}_{n_{\left(\alpha_{1}^{\lambda}\left\langle\Delta_{1}, \Delta_{2}\right\rangle\right)_{1}}} \neq \mathscr{B}_{n_{\left(\kappa_{1}^{\lambda}\left\langle\Delta_{1}, \Delta_{2}\right\rangle\right) 2}}$. Thus, there exists a one-to-one soft correspondence of $\langle f, \theta\rangle$ on to $\left\{\mathscr{B}_{n_{\left.\left(k_{1}^{\lambda} \backslash \Delta_{1}, \Delta_{2}\right\rangle\right)}}\right.$ : $\left.\left(\kappa_{1}^{\lambda}\left\langle\Delta_{1}, \Delta_{2}\right\rangle\right) \in\langle f, \theta\rangle\right\}$. Now, $\langle f, \theta\rangle$ being VS uncountable,

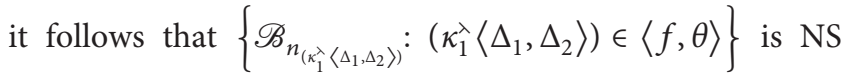
uncountable.

Theorem 14. Let $\left\langle\tilde{\pi}, \tau_{1}, \tau_{2}, \theta\right\rangle$ and $\left\langle\langle\widetilde{Y}\rangle, \mathfrak{F}_{1}, \mathfrak{F}_{2}, \theta\right\rangle$ be two VSBTS and suppose $\langle\mathfrak{f}, \theta\rangle$ be a NS continuous function, such that $\langle\mathfrak{f}, \theta\rangle:\left\langle\tilde{\pi}, \tau_{1}, \tau_{2}, \theta\right\rangle \longrightarrow\left\langle\langle\widetilde{Y}\rangle, \mathfrak{F}_{1}, \mathfrak{F}_{2}, \theta\right\rangle$ is a NS continuous function, and let $\langle\mathscr{L}, \theta\rangle \Subset\left\langle\widetilde{\pi}, \tau_{1}, \tau_{2}, \theta\right\rangle$ suppose the B.V.P. Then, safely, $\mathfrak{f}(\langle\mathscr{L}, \theta\rangle)$ has the B.V.P..

Proof. Suppose $\langle\mathscr{L}, \theta\rangle$ be an infinite VS subset of $\langle\mathfrak{f}, \theta\rangle$, so that $\langle\mathscr{L}, \theta\rangle$ contains an enumerable NS set $\left\langle\left(\kappa_{1}^{\lambda}\left\langle\Delta_{1}, \Delta_{2}\right\rangle\right)_{n}: n \in N\right\rangle$; then, there exists a enumerable NS set $\left\langle\left(\kappa_{2}^{\lambda^{\prime}}\left\langle\Delta_{1} /, \Delta_{2} /\right\rangle\right)_{n}: n \in N\right\rangle \Subset\langle\mathscr{L}, \theta\rangle \quad$ s.t., $\mathfrak{f}\left(\left(\kappa_{2}^{\lambda^{\prime}}\left\langle\Delta_{1} /, \Delta_{2} /\right\rangle\right)_{n}\right)=\left(\kappa_{1}^{\lambda}\left\langle\Delta_{1}, \Delta_{2}\right\rangle\right)_{n} . \quad\langle\mathscr{L}, \theta\rangle$ has B.V.P which implies that every infinite soft subset of $\langle\mathscr{L}, \theta\rangle$ supposes soft accumulation point belonging to $\langle\mathscr{L}, \theta\rangle$; this implies that $\left\langle\left(\kappa_{2}^{\lambda^{\prime}}\left\langle\Delta_{1} /, \Delta_{2} /\right\rangle\right)_{n}: n \in N\right\rangle$ has soft vague limit point, say, $\left(\kappa_{2}^{\lambda^{\prime}}\left\langle\Delta_{1} /, \Delta_{2} /\right\rangle\right)_{0} \in\langle\mathscr{L}, \theta\rangle$ implies that the limit of soft sequence $\left\langle\left(\kappa_{2}^{\prime}\left\langle\Delta_{1} /, \Delta_{2} /\right\rangle\right)_{n}: n \in N\right\rangle \quad$ is $\left(\kappa_{2}^{\lambda^{\prime}}\left\langle\Delta_{1} /, \Delta_{2} /\right\rangle\right)_{0} \in\langle\mathscr{L}, \theta\rangle \Rightarrow\left(\kappa_{2}^{\lambda^{\prime}}\left\langle\Delta_{1} /, \Delta_{2} /\right\rangle\right)_{n} \longrightarrow\left(\kappa_{2}^{\lambda^{\prime}}\left\langle\Delta_{1} /\right.\right.$, $\left.\left.\Delta_{2} /\right\rangle\right)_{0} \in\langle\mathscr{L}, \theta\rangle . f$ is soft continuous implying that it is soft continuous. Furthermore, $\left(\kappa_{2}^{\lambda^{\prime}}\left\langle\Delta_{1} /, \Delta_{2} /\right\rangle\right)_{n} \longrightarrow\left(\kappa_{2}^{\lambda^{\prime}}\right.$ $\left.\left\langle\Delta_{1} /, \Delta_{2}\right\rangle\right)_{0} \in\langle\mathscr{L}, \theta\rangle \Rightarrow \mathfrak{f}\left(\left(\kappa_{2}^{\lambda^{\prime}}\left\langle\Delta_{1} /, \Delta_{2} /\right\rangle\right)_{n}\right) \longrightarrow \mathfrak{F}\left(\left(\left(\kappa_{2}^{\lambda^{\prime}}\left\langle\Delta_{1} /\right.\right.\right.\right.$, $\left.\left.\left.\left.\Delta_{2} /\right\rangle\right)_{0}\right)\right) \in \mathfrak{f} \quad(\langle\mathscr{L}, \theta\rangle) \Rightarrow\left(\kappa_{1}^{\lambda} \quad\left\langle\Delta_{1}, \Delta_{2}\right\rangle\right)_{n} \longrightarrow \quad \mathfrak{f}\left(\left(\kappa_{2}^{\lambda^{\prime}}\left\langle\Delta_{1} /\right.\right.\right.$, $\left.\left.\left.\Delta_{2} /\right\rangle\right)_{0}\right) \in \mathfrak{f}(\langle\mathscr{L}, \theta\rangle)$ implies that limit of a soft sequence $\left\langle\left(\kappa_{1}^{\lambda}\left\langle\Delta_{1}, \Delta_{2}\right\rangle\right)_{n}: n \in N\right\rangle$ is $\mathfrak{f}\left(\left(\kappa_{2}^{\prime}\left\langle\Delta_{1} /, \Delta_{2} /\right\rangle\right)_{0}\right) \in \mathfrak{f}(\langle\mathscr{L}, \theta\rangle)$, 
implying that limit of a soft sequence $\left\langle\left(\kappa_{1}^{\lambda}\left\langle\Delta_{1}, \Delta_{2}\right\rangle\right)_{n}: n \in N\right\rangle$ is $\mathfrak{f}\left(\left(\kappa_{2}^{\lambda^{\prime}}\left\langle\Delta_{1} /, \Delta_{2} /\right\rangle\right)_{0}\right) \quad \in \mathfrak{f}, \theta(\langle\mathscr{L}$, $\theta\rangle)$. Finally, we have shown that there exists an infinite soft subset $\left\langle\left(\kappa_{1}^{\lambda}\left\langle\Delta_{1}, \Delta_{2}\right\rangle\right)_{n}: n \in N\right\rangle$ of $\mathfrak{f}(\langle\mathscr{L}, \theta\rangle)$ containing a limit point $\mathfrak{f}\left(\left(\kappa_{2}^{\lambda^{\prime}}\left\langle\Delta_{1} /, \Delta_{2} /\right\rangle\right)_{0}\right) \in \mathfrak{f}(\langle\mathscr{L}, \theta\rangle)$. This guarantees that $\mathfrak{f}(\langle\mathscr{L}, \theta\rangle)$ has B.V.P.

Theorem 15. Let $\left\langle\tilde{\pi}, \tau_{1}, \tau_{2}, \theta\right\rangle$ VSBTS and let $\left(\kappa_{1}^{\lambda}\left\langle\widetilde{\Delta_{1}, \Delta_{2}}\right\rangle\right)_{n}$ be a VS sequence in $\left\langle\tilde{\pi}, \tau_{1}, \tau_{2}, \theta\right\rangle$, such that it converges to a point $\left(\kappa_{1}^{\lambda}\left\langle\Delta_{1}, \Delta_{2}\right\rangle\right)_{0}$; then, the soft set $\langle g, \theta\rangle$ consisting of the points $\left(\kappa_{1}^{\lambda}\left\langle\Delta_{1}, \Delta_{2}\right\rangle\right)_{n_{0}}$ and $\left(\kappa_{1}^{\lambda}\left\langle\Delta_{1}, \Delta_{2}\right\rangle\right)_{n}(n=1,2,3, \ldots)$ is soft VSB compact.

Proof. Given $\left\langle\tilde{\pi}, \tau_{1}, \tau_{2}, \theta\right\rangle$ VSBTS and let $\left(\kappa_{1}^{\lambda}\left\langle\widetilde{\Delta_{1}}, \Delta_{2}\right\rangle\right)_{n}$ be a VS sequence in $\left\langle\tilde{\pi}, \tau_{1}, \tau_{2}, \theta\right\rangle$, such that it converges to a point $\left(\kappa_{1}^{\lambda}\left\langle\Delta_{1}, \Delta_{2}\right\rangle\right)_{n_{0}}$, that is, $\left(\kappa_{1}^{\lambda}\left\langle\widetilde{\Delta_{1}, \Delta_{2}}\right\rangle\right)_{n} \longrightarrow\left(\kappa_{1}^{\lambda}\left\langle\Delta_{1}\right.\right.$, $\left.\left.\Delta_{2}\right\rangle\right)_{n_{0}} \in \tilde{\pi}$. Let $\quad\langle g, \theta\rangle=$ $\left[\begin{array}{c}\kappa_{1}^{\lambda}\left\langle\widetilde{\Delta_{1}}, \Delta_{2}\right\rangle,\left(\kappa_{1}^{\lambda}\left\langle\widetilde{\Delta_{1}}, \Delta_{2}\right\rangle\right)_{2},\left(\kappa_{1}^{\lambda}\left\langle\widetilde{\Delta_{1}}, \Delta_{2}\right\rangle\right)_{3}, \\ \left.\left(\kappa_{1}^{\lambda}\left\langle\widetilde{\Delta_{1}}, \Delta_{2}\right\rangle\right)_{4},\left(\kappa_{1}^{\lambda} \widetilde{\left\langle\Delta_{1}\right.}, \Delta_{2}\right\rangle\right)_{5}, \\ \left(\kappa_{1}^{\lambda}\left\langle\widetilde{\Delta_{1}, \Delta_{2}}\right\rangle\right)_{6},\left(\kappa_{1}^{\lambda}\left\langle\widetilde{\Delta_{1}, \Delta_{2}}\right\rangle\right)_{7}, \ldots\end{array}\right]$. Let $\left\{\left\langle\widetilde{S}, \theta_{\alpha}\right\rangle:\right.$ $\alpha \in \Delta\}$ be VS*b open covering of $\langle g, \theta\rangle$, so that $\langle g, \theta\rangle \Subset \widetilde{U}\left\{\left\langle\widetilde{S}, \theta_{\alpha}\right\rangle: \alpha \in \Delta\right\},\left(\kappa_{1}^{\lambda}\left\langle\Delta_{1}, \Delta_{2}\right\rangle\right)_{n_{0}} \in\langle g, \theta\rangle$ implies that $\exists \alpha_{0} \in \Delta$, such that $\left(\kappa_{1}^{\lambda}\left\langle\Delta_{1}, \Delta_{2}\right\rangle\right)_{n_{0}} \in\left\langle\mathfrak{S}, \theta_{\alpha_{0}}\right\rangle$. According to the definition of soft convergence, $\left(\kappa_{1}^{\lambda}\left\langle\Delta_{1}, \Delta_{2}\right\rangle\right)_{n_{0}} \in\left\langle\mathfrak{S}, \theta_{\alpha_{0}}\right\rangle \in\left(\tilde{\pi}, \tau_{1}, \tau_{2}, \theta\right)$ implies that there exists $n_{0} \in N$ s.t. $n \geq n_{0}$ and $\left(\kappa_{1}^{\lambda}\left\langle\Delta_{1}, \Delta_{2}\right\rangle\right)_{n} \in\left\langle\mathfrak{S}, \theta_{\alpha_{0}}\right\rangle$. Evidently, $\left\langle\widetilde{S}, \theta_{\alpha_{0}}\right\rangle$ contains the points $\left(\kappa_{1}^{\lambda}\left\langle\Delta_{1}\right.\right.$, $\left.\left.\Delta_{2}\right\rangle\right)_{n_{0}},\left(\kappa_{1}^{\lambda}\left\langle\Delta_{1}, \Delta_{2}\right\rangle\right)_{n_{0+1}},\left(\kappa_{1}^{\lambda}\left\langle\Delta_{1}, \Delta_{2}\right\rangle\right)_{n_{0+2}}$,

$$
\left(\kappa_{1}^{\lambda}\left\langle\Delta_{1}, \Delta_{2}\right\rangle\right)_{n_{0+3}},\left(\kappa_{1}^{\lambda}\left\langle\Delta_{1}, \Delta_{2}\right\rangle\right), \ldots
$$

$\left(\kappa_{1}^{\lambda}\left\langle\Delta_{1}, \Delta_{2}\right\rangle\right)_{n_{0+n}}$. Look carefully at the points and train them in a way as $\left(\kappa_{1}^{\lambda}\left\langle\Delta_{1}, \Delta_{2}\right\rangle\right)_{1},\left(\kappa_{1}^{\lambda}\left\langle\Delta_{1}, \Delta_{2}\right\rangle\right)_{2},\left(\kappa_{1}^{\lambda}\left\langle\Delta_{1}, \Delta_{2}\right\rangle\right)_{3}$,

$$
\left(\kappa_{1}^{\lambda}\left\langle\Delta_{1}, \Delta_{2}\right\rangle\right)_{4}, \ldots
$$

$\left(\kappa_{1}^{\lambda}\left\langle\Delta_{1}, \Delta_{2}\right\rangle\right)_{n}$, generating a finite soft set. Let $1 \leq n_{0-1}$. Then, $\left(\kappa_{1}^{\lambda}\left\langle\Delta_{1}, \Delta_{2}\right\rangle\right)_{i} \in\langle g, \theta\rangle$. For this, $i,\left(\kappa_{1}^{\lambda}\left\langle\Delta_{1}, \Delta_{2}\right\rangle\right)_{i} \in\langle g, \theta\rangle$. Hence, there exists $\alpha_{i} \in \Delta$, such that $\left(\kappa_{1}^{\lambda}\left\langle\Delta_{1}, \Delta_{2}\right\rangle\right)_{i} \in\left\langle\mathfrak{S}, \theta_{\alpha_{i}}\right\rangle$. Evidently, $g, \theta \Subset \cup_{r=0}^{n_{0-1}}\left\langle\widetilde{\mathfrak{S}, \theta_{\alpha_{i}}}\right\rangle$. This shows that $\left\{\left\langle\mathfrak{S}, \theta_{\alpha_{i}}\right\rangle: 0 \leq n_{0-1}\right\}$ is VS* $*_{b}$ open cover of $\langle\mathrm{g}, \theta\rangle$. Thus, an arbitrary VS $*_{b}$ open cover $\left\{\left\langle\widetilde{\Phi}, \theta_{\alpha}\right\rangle: \alpha \in \Delta\right\}$ of $\langle\mathrm{g}, \theta\rangle$ is reducible to a finite VS subcover $\left\{\left\langle\widetilde{S}, \theta_{\alpha i}\right\rangle: i=0,1,2,3, \ldots n_{0-1}\right\}$, and it follows that $\langle\mathrm{g}, \theta\rangle$ is soft VSB $*_{b}$ compact.

Theorem 16. If $\left(\tilde{\pi}, \tau_{1}, \tau_{2}, \theta\right)$ VSBTS, it has the characteristics of NS $*_{b}$ sequentially compactness. Then, $\left\langle\tilde{\pi}, \tau_{1}, \tau_{2}, \theta\right\rangle$ is safely VSB* $*_{b}$ countably compact.

Proof. Let VSB $*_{b}$, and let $\langle\rho, \theta\rangle$ be finite soft subset of

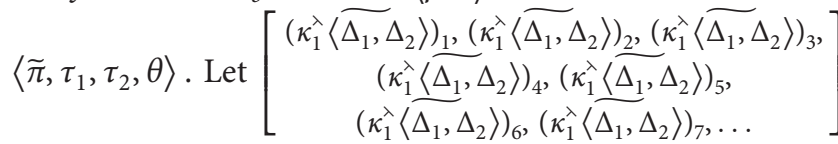
be a soft sequence of soft points of $\langle\rho, \theta\rangle$. Then, $\langle\rho, \theta\rangle$ being finite, at least one of the elements. In $\langle\rho, \theta\rangle$, say $\left(\kappa_{1}^{\lambda}\left\langle\widetilde{\Delta_{1}, \Delta_{2}}\right\rangle\right)_{0}$ must be duplicated in an infinite number of times in the VS sequence. Hence,

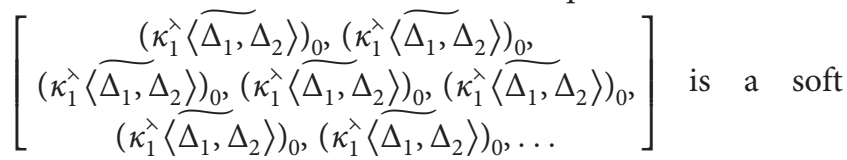
subsequence of $\left\langle\left(\kappa_{1}^{\lambda}\left\langle\widetilde{\Delta_{1}, \Delta_{2}}\right\rangle\right)_{n}\right\rangle$, such that it is soft constant sequence and repeatedly constructed by single soft number $\left(\kappa_{1}^{\lambda}\left\langle\widetilde{\Delta_{1}, \Delta_{2}}\right\rangle\right)_{0}$, and we know that a soft constant sequence converges on itself. So, it converges to $\left(\kappa_{1}^{\lambda}\left\langle\widetilde{\Delta_{1}, \Delta_{2}}\right\rangle\right)_{0}$, which belongs to $\langle\rho, \theta\rangle$. Hence, $\langle\rho, \theta\rangle$ is soft sequentially $\operatorname{VSB} *_{b}$ compact.

Theorem 17. Let $\left\langle\tilde{\pi}, \tau_{1}, \tau_{2}, \theta\right\rangle$ VSBTS and $\left(\langle\widetilde{Y}\rangle, \mathfrak{\mho}_{1}, \mathfrak{F}_{2}, \theta\right)$ be another VSBTS. Let $\langle f, \theta\rangle$ be a soft continuous mapping of a soft vague sequentially compact $N S *_{b}$ space $\left\langle\tilde{\pi}, \tau_{1}, \tau_{2}, \theta\right\rangle$ into $\left(\langle\widetilde{Y}\rangle, \mathfrak{F}_{1}, \widetilde{F}_{2}, \theta\right)$. Then, $\langle f, \theta\rangle\left\langle\widetilde{\pi}, \tau_{1}, \tau_{2}, \theta\right\rangle$ is $V S B *_{b}$ sequentially compact.

Proof. Given $\left\langle\widetilde{\pi}, \tau_{1}, \tau_{2}, \theta\right\rangle$ VSBTS and $\left(\langle\widetilde{Y}\rangle, \mathfrak{F}_{1}, \mathfrak{F}_{2}, \theta\right)$ be another VSBTS. Let $\langle f, \theta\rangle$ be a soft continuous mapping of a VSB sequentially compact space $\left(\tilde{\pi}, \tau_{1}, \tau_{2}, \theta\right)$ into $\left(\langle\widetilde{Y}\rangle, \mathfrak{F}_{1}, \mathfrak{F}_{2}, \theta\right)$; then, we have to prove $\langle f, \theta\rangle\left\langle\widetilde{\pi}, \tau_{1}, \tau_{2}, \theta\right\rangle$ VS sequentially. For this, we proceed as let

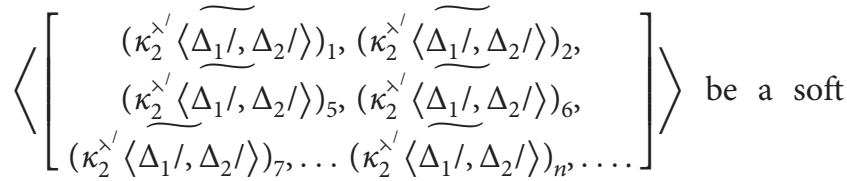
sequence of VS points in $\langle f, \theta\rangle\left\langle\widetilde{\pi}, \tau_{1}, \tau_{2}, \theta\right\rangle$. Then, for each $n \in N, \quad$ there exists

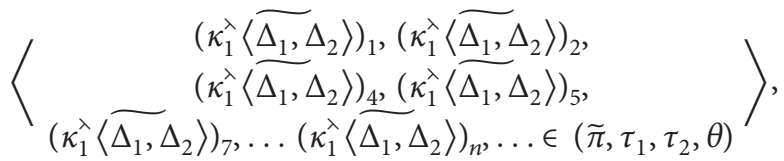
such that $\langle f, \theta\rangle\left[\left(\left(\widetilde{\kappa_{1}^{\lambda}\left\langle\Delta_{1}\right.}, \quad \Delta_{2}\right\rangle\right)_{1},\left(\kappa_{1}^{\lambda}\left\langle\widetilde{\Delta_{1}, \Delta_{2}}\right\rangle\right)_{2}, \widetilde{\left(\kappa_{1}^{\lambda}\right.}\right.$ $\left.\left.\left\langle\Delta_{1}, \Delta_{2}\right\rangle\right)_{4},\left(\kappa_{1}^{\lambda}\left\langle\widetilde{\Delta_{1}, \Delta_{2}}\right\rangle\right)_{5}, \widetilde{\left(\kappa_{1}^{\lambda}\right.} \quad\left\langle\Delta_{1}, \Delta_{2}\right\rangle\right)_{7}, \ldots\left(\kappa_{1}^{\lambda}\left\langle\widetilde{\Delta_{1}, \Delta_{2}}\right\rangle\right)_{n}$, $\left.\left.\left.\ldots \in\left(\tilde{\pi}, \tau_{1}, \tau_{2}, \theta\right)\right)\right]=\left\langle\left[\widetilde{\left(\kappa_{2}\right.} \underline{\left\langle\Delta_{1}^{\prime} /, \Delta_{2}\right\rangle}\right)_{1}, \widetilde{\left(\kappa_{2}^{\lambda^{\prime}}\right.}\left\langle\Delta_{1} /, \Delta_{2}\right\rangle\right)\right)_{2}, \widetilde{\left(\kappa_{2}^{\lambda^{\prime}}\right.}$ $\left.\left.\left.\left\langle\Delta_{1}, \Delta_{2} /\right\rangle\right)_{3}, \widetilde{\left(\kappa_{2}^{\lambda^{\prime}}\right.} \quad\left\langle\Delta_{1} /, \Delta_{2} l\right\rangle\right)_{4}, \widetilde{\left(\kappa_{2}^{\lambda^{\prime}}\right.} \quad\left\langle\Delta_{1} /, \Delta_{2} /\right\rangle\right)_{6},\left(\kappa_{2}^{\lambda^{\prime}}\left\langle\Delta_{1} /\right.\right.$,

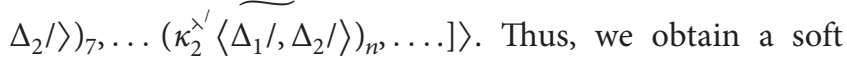

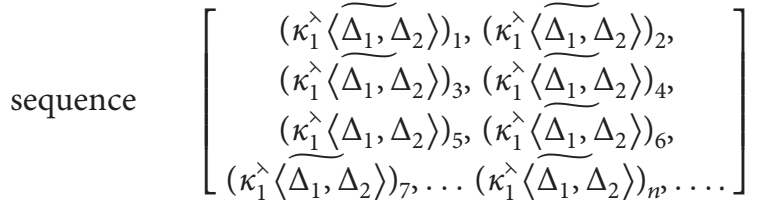
in $\left\langle\tilde{\pi}, \tau_{1}, \tau_{2}, \theta\right\rangle$. But $\left\langle\widetilde{\pi}, \tau_{1}, \tau_{2}, \theta\right\rangle$ being soft sequentially VSB $*_{b}$ compact, there is a VS subsequence $\left\langle\left(\kappa_{1}^{\lambda}\left\langle\widetilde{\Delta_{1}, \Delta_{2}}\right\rangle\right)_{n_{i}}\right\rangle$ of $\left\langle\left(\kappa_{1}^{\lambda}\left\langle\widetilde{\Delta_{1}, \Delta_{2}}\right\rangle\right)_{n_{i}}\right\rangle$, such that $\left\langle\left(\kappa_{1}^{\lambda}\left\langle\widetilde{\Delta_{1}, \Delta_{2}}\right\rangle\right)_{n_{i}}\right\rangle \longrightarrow$ $\left(\kappa_{1}^{\lambda}\left\langle\Delta_{1}, \Delta_{2}\right\rangle\right) \in\left\langle\tilde{\pi}, \tau_{1}, \tau_{2}, \theta\right\rangle$. So, by $\mathrm{VS} *_{b}$ continuity of $\langle f, \theta\rangle,\left\langle\left(\kappa_{1}^{\lambda}\left\langle\widetilde{\Delta_{1}, \Delta_{2}}\right\rangle\right)_{n_{i}}\right\rangle \longrightarrow\left(\kappa_{1}^{\lambda}\left\langle\widetilde{\Delta_{1}, \Delta_{2}}\right\rangle \theta\right)$ implies that $\left.\langle f, \theta\rangle\left(\left\langle\left(\widetilde{\kappa_{1}^{\lambda}\left\langle\Delta_{1}\right.}, \quad \Delta_{2}\right\rangle\right)_{n_{i}}\right\rangle\right) \longrightarrow\langle f, \theta\rangle\left(\left\langle\left(\kappa_{1}^{\lambda}\left\langle\widetilde{\Delta_{1}, \Delta_{2}}\right\rangle\right)_{n}\right\rangle\right) \epsilon$ $\langle f, \theta\rangle\left\langle\tilde{\pi}, \tau_{1}, \tau_{2}, \theta\right\rangle$. Thus, $\langle f, \theta\rangle\left(\left\langle\left(\kappa_{2}^{\prime}\left\langle\widetilde{\Delta_{1} /, \Delta_{2}} /\right\rangle\right)_{n_{i}}\right\rangle\right)$ is a 


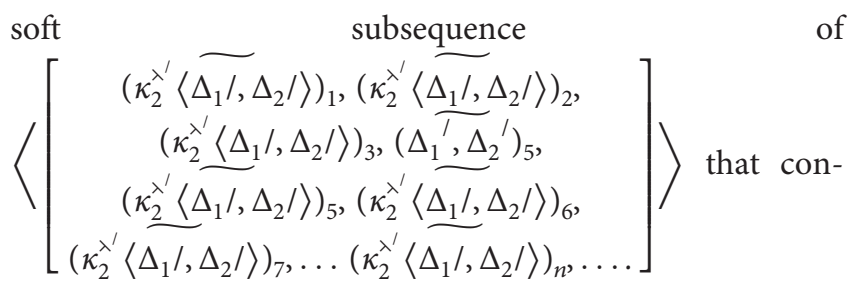
verges to $(\langle f, \theta\rangle)\left(\tilde{\kappa}_{1}\right)$ in $\langle f, \theta\rangle\left\langle\tilde{\pi}, \tau_{1}, \tau_{2}, \theta\right\rangle$. Hence, $\langle f, \theta\rangle\left\langle\tilde{\pi}, \tau_{1}, \tau_{2}, \theta\right\rangle$ is $\mathrm{VS} *_{b}$ sequentially compact.

Theorem 18. Let $\left\langle\tilde{\pi}, \tau_{1}, \tau_{2}, \theta\right\rangle V S B T S$, and suppose $\langle f, \theta\rangle,\langle g, \theta\rangle$ be two NS continuous function on VS BTS $\left\langle\widetilde{\pi}, \tau_{1}, \tau_{2}, \theta\right\rangle$ into VSBTS $\left\langle\widetilde{Y}, \mathfrak{F}_{1}, \mathfrak{F}_{2}, \theta\right\rangle$, which is VSB*b Hausdorff. Then, soft set $\left\{\left(\kappa_{1}^{\lambda}\left\langle\Delta_{1}, \Delta_{2}\right\rangle\right) \in \tilde{\pi}\right.$ : $\left.(f)\left(\left(\kappa_{1}^{\lambda}\left\langle\Delta_{1}, \Delta_{2}\right\rangle\right)\right)=(g)\left(\left(\kappa_{1}^{\lambda}\left\langle\Delta_{1}, \Delta_{2}\right\rangle\right)\right)\right\}$ is VS* closed of $\left\langle\widetilde{\pi}, \tau_{1}, \tau_{2}, \theta\right\rangle$.

Proof. Let $\left\{\left(\kappa_{1}^{\lambda}\left\langle\Delta_{1}, \Delta_{2}\right\rangle\right) \in \tilde{\pi}:(f)\left(\left(\kappa_{1}^{\lambda}\left\langle\Delta_{1}, \Delta_{2}\right\rangle\right)\right)=(g)\left(\left(\kappa_{1}^{\lambda}\right.\right.\right.$ $\left.\left.\left.\left\langle\Delta_{1}, \Delta_{2}\right\rangle\right)\right)\right\}$ is a VS set of function. If $\left\{\left(\kappa_{1}^{\lambda}\left\langle\Delta_{1}, \Delta_{2}\right\rangle\right) \epsilon\right.$ $\left.\tilde{\pi}:(f)\left(\left(\kappa_{1}^{\lambda}\left\langle\Delta_{1}, \Delta_{2}\right\rangle\right)\right)=(g)\left(\kappa_{1}\right)\right\}^{c}=\tilde{\varnothing}$, it is clearly VS $*_{b}$ open, and therefore, $\left\{\left(\kappa_{1}^{\lambda}\left\langle\Delta_{1}, \Delta_{2}\right\rangle\right) \in \tilde{\pi}:(f)\left(\left(\kappa_{1}^{\lambda}\left\langle\Delta_{1}, \Delta_{2}\right\rangle\right)\right)=\right.$ ( $\left.g)\left(\left(\kappa_{1}^{\lambda}\left\langle\Delta_{1}, \Delta_{2}\right\rangle\right)\right)\right\}$ is VS $*_{b}$ closed, that is, nothing is proved in this case. Let us consider the case when $\left\{\left(\kappa_{1}^{\lambda}\left\langle\Delta_{1}, \Delta_{2}\right\rangle\right) \in \tilde{\pi}:(f) \quad\left(\left(\kappa_{1}^{\lambda}\left\langle\Delta_{1}, \Delta_{2}\right\rangle\right)\right)=(g)\left(\left(\kappa_{1}^{\lambda}\left\langle\Delta_{1}\right.\right.\right.\right.$, $\left.\left.\left.\left.\Delta_{2}\right\rangle\right)\right)\right\}^{c} \neq\left(\kappa_{1}^{\lambda}\left\langle\Delta_{1}, \Delta_{2}\right\rangle\right)$. Let $\quad \rho \in\left\{\left(\kappa_{1}^{\lambda}\left\langle\Delta_{1}, \Delta_{2}\right\rangle\right) \in \tilde{\widetilde{\pi}}\right.$ : (f) $\left.\left(\left(\kappa_{1}^{\lambda}\left\langle\Delta_{1}, \Delta_{2}\right\rangle\right)\right)=(g)\left(\left(\kappa_{1}^{\lambda}\left\langle\Delta_{1}, \Delta_{2}\right\rangle\right)\right)\right\}^{c}$. Then, $\rho$ does not belong to $\left\{\left(\kappa_{1}^{\lambda}\left\langle\Delta_{1}, \Delta_{2}\right\rangle\right) \in \tilde{\pi}:(f)\left(\left(\kappa_{1}^{\lambda}\left\langle\Delta_{1}, \Delta_{2}\right\rangle\right)\right)=(g)\left(\kappa_{1}\right)\right\}$. Result in $(f)(\rho) \neq(g)(\rho)$. Now, $\left(\langle\widetilde{Y}\rangle, \mathfrak{F}_{1}, \mathfrak{\mho}_{2}, \theta\right)$ be VSB $*_{b}$ Hausdorff space, so there exists VS $*_{b}$ open sets $\langle g, \theta\rangle$, $\langle\mathfrak{H}, \theta\rangle$ of $(f)(\rho)$ and $(g)(\rho)$, respectively, such that $\langle g, \theta\rangle$ and $\langle\mathfrak{S}, \theta\rangle$, and these NS sets are mutually disjoint. By soft continuity of $\langle f, \theta\rangle,\langle g, \theta\rangle,\langle f, \theta\rangle^{-1}$ as well as $\langle g, \theta\rangle^{-1}$ is $\mathrm{VS} * *_{b}$ open neighborhood of $\rho$, and therefore, so is $\langle f, \theta\rangle^{-1} \widetilde{\cap}\langle g, \theta\rangle^{-1} \quad$ contained in $\left\{\left(\kappa_{1}^{\lambda}\left\langle\Delta_{1}, \Delta_{2}\right\rangle\right) \in \tilde{\pi}\right.$ : $\left.(f)\left(\left(\kappa_{1}^{\lambda}\left\langle\Delta_{1}, \Delta_{2}\right\rangle\right)\right)=(g)\left(\left(\kappa_{1}^{\lambda}\left\langle\Delta_{1}, \Delta_{2}\right\rangle\right)\right)\right\}$ for $\left(\kappa_{1}^{\lambda}\left\langle\Delta_{1}, \Delta_{2}\right\rangle\right) \epsilon$ $\left(\langle f, \theta\rangle^{-1} \frac{\pi}{\cap}\langle g, \theta\rangle^{-1}\right) \Rightarrow(f)\left(\left(\kappa_{1}^{\lambda}\left\langle\Delta_{1}, \Delta_{2}\right\rangle\right)\right) \in\langle g, \theta\rangle \quad$ and $(g)(f)\left(\left(\kappa_{1}^{\lambda}\left\langle\Delta_{1}, \Delta_{2}\right\rangle\right)\right) \neq(g)\left(\left(\kappa_{1}^{\lambda}\left\langle\Delta_{1}, \Delta_{2}\right\rangle\right)\right)$ because $\langle g, \theta\rangle$ and $\langle\mathfrak{H}, \theta\rangle$ are mutually exclusive. This implies that $\kappa_{1}$ does not belong to $\left\{\left(\kappa_{1}^{\lambda}\left\langle\Delta_{1}, \Delta_{2}\right\rangle\right) \in \tilde{\pi}:(f)\left(\left(\kappa_{1}^{\lambda}\left\langle\Delta_{1}\right.\right.\right.\right.$, $\left.\left.\left.\left.\Delta_{2}\right\rangle\right)\right)=(g)\left(\left(\kappa_{1}^{\lambda}\left\langle\Delta_{1}, \Delta_{2}\right\rangle\right)\right)\right\}$. Therefore, $\rho \in(f)^{-1}(\langle g, \theta\rangle)$ $\widetilde{\cap}(g)^{-1} \quad(\langle g, \theta\rangle) \Subset\left\{\kappa_{1}^{\lambda}\left\langle\Delta_{1}, \quad \Delta_{2}\right\rangle \in \tilde{\pi}:(f)\left(\kappa_{1}^{\lambda}\left\langle\Delta_{1}, \Delta_{2}\right\rangle\right)=\right.$ (g) $\left.\left(\kappa_{1}^{\lambda}\left\langle\Delta_{1}, \Delta_{2}\right\rangle\right)\right\}^{c}$. This shows that $\left\{\kappa_{1}^{\lambda}\left\langle\Delta_{1}, \Delta_{2}\right\rangle \in\right.$ $\left.\tilde{\pi}:(f)\left(\kappa_{1}^{\lambda}\left\langle\Delta_{1}, \Delta_{2}\right\rangle\right)=(g)\left(\kappa_{1}^{\lambda}\left\langle\Delta_{1}, \Delta_{2}\right\rangle\right)\right\}^{c}$ is neighborhood of each of its points. So, $\left\{\kappa_{1}^{\lambda}\left\langle\Delta_{1}, \Delta_{2}\right\rangle \in \tilde{\pi}\right.$ : $(f)\left(\kappa_{1}^{\lambda}\left\langle\Delta_{1}, \Delta_{2}\right\rangle\right)=$ $\left.(g)\left(\kappa_{1}^{\lambda}\left\langle\Delta_{1}, \Delta_{2}\right\rangle\right)\right\}^{c} \quad \mathrm{NS} *_{b} \quad$ open, and hence, $\left\{\kappa_{1}^{\lambda}\left\langle\Delta_{1}, \Delta_{2}\right\rangle \in \tilde{\pi}:(f)\left(\kappa_{1}^{\lambda}\left\langle\Delta_{1}, \Delta_{2}\right\rangle\right)=(g)\left(\kappa_{1}^{\lambda}\left\langle\Delta_{1}, \Delta_{2}\right\rangle\right)\right\} \quad$ is $\mathrm{VS} *_{b}$ closed.

Theorem 19. Let $\left\langle\tilde{\pi}, \tau_{1}, \tau_{2}, \theta\right\rangle$ VSBTS, such that it is VSB* $*_{b}$ Hausdorff space, and let (f) be soft continuous function of $\left(\tilde{\pi}, \tau_{1}, \tau_{2}, \theta\right)$ into itself. Then, the VS set of fixed points under (f) is a VSB* $*_{b}$ closed set.

Proof. Let $\delta=\left\{(f)\left(\left(\kappa_{1}^{\lambda}\left\langle\Delta_{1}, \Delta_{2}\right\rangle\right)\right)=\left(\kappa_{1}^{\lambda}\left\langle\Delta_{1}, \Delta_{2}\right\rangle\right)\right\}$. If $\delta^{c}=\tilde{\varnothing}$, then is $\mathrm{VS} *_{b}$ open, and therefore, $\left\{(f)\left(\left(\kappa_{1}^{\lambda}\left\langle\Delta_{1}\right.\right.\right.\right.$, $\left.\left.\left.\left.\Delta_{2}\right\rangle\right)\right)=\left(\kappa_{1}^{\lambda}\left\langle\Delta_{1}, \Delta_{2}\right\rangle\right)\right\} \mathrm{VS} *_{b}$ closed. So, let $\left\{(f)\left(\left(\kappa_{1}^{\lambda}\left\langle\Delta_{1}\right.\right.\right.\right.$,
$\left.\left.\left.\left.\Delta_{2}\right\rangle\right)\right)=\left(\kappa_{1}^{\lambda}\left\langle\Delta_{1}, \Delta_{2}\right\rangle\right)\right\}^{c} \neq \tilde{\varnothing}, \quad$ and $\quad$ let $\quad \kappa_{2}^{\lambda^{\prime}}\left\langle\Delta_{1} /, \Delta_{2} /\right\rangle \in$ $\left\{(f)\left(\left(\kappa_{1}^{\lambda}\left\langle\Delta_{1}, \Delta_{2}\right\rangle\right)\right)=\left(\kappa_{1}^{\lambda}\left\langle\Delta_{1}, \Delta_{2}\right\rangle\right)\right\}^{c}$. Then, $\kappa_{2}^{\lambda^{\prime}}\left\langle\Delta_{1} /, \Delta_{2} /\right\rangle$ does not belong to $\left\{(f)\left(\kappa_{1}^{\lambda}\left\langle\Delta_{1}, \Delta_{2}\right\rangle\right)=\kappa_{1}^{\lambda}\left\langle\Delta_{1}, \Delta_{2}\right\rangle\right\} \varepsilon$, and therefore, (f) $\left(\kappa_{2}{ }^{\lambda^{\prime}} \Delta_{1}{ }^{\prime}, \Delta_{2}{ }^{\prime}\right) \neq \kappa_{2}{ }^{\lambda^{\prime}}{ }_{\Delta_{1}{ }^{\prime}, \Delta_{2}{ }^{\prime}}$. Now, $\kappa_{2}^{\lambda^{\prime}}\left\langle\Delta_{1} /, \Delta_{2} /\right\rangle$, $(f)\left(\kappa_{2}^{\lambda^{\prime}}\left\langle\Delta_{1} /, \Delta_{2} /\right\rangle\right)$ being two distinct points of the $\operatorname{VSB} *_{b}$ Hausdorff space $\left\langle\tilde{\pi}, \tau_{1}, \tau_{2}, \theta\right\rangle$, there exists $\mathrm{VS} *_{b}$ open sets $\langle g, \theta\rangle, \quad\langle\mathfrak{H}, \theta\rangle, \quad$ such that $\kappa_{2}^{\lambda^{\prime}}\left\langle\Delta_{1} /, \Delta_{2} /\right\rangle \in\langle g, \theta\rangle,(f)$ $\left(\kappa_{2}^{\lambda^{\prime}}\left\langle\Delta_{1} /, \Delta_{2} /\right\rangle\right) \in\langle\mathfrak{H}, \theta\rangle$ and $\langle g, \theta\rangle,\langle\mathfrak{H}, \theta\rangle$ are disjoints. Also, by the VS continuity of $(\mathrm{f}),(f)^{-1}(H, \theta)$ is a $\mathrm{VS} *_{b}$-open set. We pretend that $\langle g, \theta\rangle \widetilde{\cap}(f)^{-1}(\langle\mathfrak{H}, \theta\rangle)$ $\Subset\left\{(f)\left(\left(\kappa_{1}^{\lambda}\left\langle\Delta_{1}, \Delta_{2}\right\rangle\right)\right)=\left(\kappa_{1}^{\lambda}\left\langle\Delta_{1}, \Delta_{2}\right\rangle\right)\right\}^{c}$. Since $\mu \in\langle g, \theta\rangle \widetilde{\cap}$ $(f)^{-1}(\langle\mathfrak{H} \quad, \theta\rangle) \Rightarrow \mu \in\langle g, \theta\rangle, \mu \in(f)^{-1} \Rightarrow \mu \in\langle g, \quad \theta\rangle,(f)$ $(\mu) \in\langle\mathfrak{H}, \theta\rangle \Rightarrow \mu \neq(f)(\mu) .\langle g, \theta\rangle \widetilde{\cap}\langle\mathfrak{H}, \theta\rangle=\tilde{\varnothing}$ implies that $\mu$ does not belong to $\left\{(f)\left(\kappa_{1}^{\lambda}\left\langle\Delta_{1}, \Delta_{2}\right\rangle\right)=\right.$ $\left.\left(\kappa_{1}^{\lambda}\left\langle\Delta_{1}, \Delta_{2}\right\rangle\right)\right\} \Rightarrow \mu \in\left\{(f)\left(\kappa_{1}^{\lambda}\left\langle\Delta_{1}, \Delta_{2}\right\rangle\right)=\kappa_{1}^{\lambda}\left\langle\Delta_{1}, \Delta_{2}\right\rangle\right\}^{c}$.

Therefore, $\quad \kappa_{2}^{\lambda^{\prime}}\left\langle\Delta_{1} /, \Delta_{2} /\right\rangle \in\langle g, \theta\rangle \widetilde{\cap}(f)^{-1}(\langle\mathfrak{H}, \theta\rangle) \Subset\{(f)$ $\left.\left(\kappa_{1}^{\lambda}\left\langle\Delta_{1}, \Delta_{2}\right\rangle\right)=\left(\kappa_{1}^{\lambda}\left\langle\Delta_{1}, \Delta_{2}\right\rangle\right)\right\}^{c}$. Thus, $\left\{(f)\left(\left(\kappa_{1}^{\lambda}\left\langle\Delta_{1}, \Delta_{2}\right\rangle\right)\right)=\right.$ $\left.\left(\kappa_{1}^{\lambda}\left\langle\Delta_{1}, \Delta_{2}\right\rangle\right)\right\}^{c}$ is the NS nhd of each of its points. So, $\left\{(f)\left(\left(\kappa_{1}^{\lambda}\left\langle\Delta_{1}, \Delta_{2}\right\rangle\right)\right)=\left(\kappa_{1}^{\lambda}\left\langle\Delta_{1}, \Delta_{2}\right\rangle\right)\right\}^{c}$ is $\mathrm{NS} *_{b}$ open, and hence, $\quad\left\{(f)\left(\left(\kappa_{1}^{\lambda}\left\langle\Delta_{1}, \Delta_{2}\right\rangle\right)\right)=\left(\kappa_{1}^{\lambda}\left\langle\Delta_{1}, \Delta_{2}\right\rangle\right)\right\}$ is $\operatorname{VSB} *_{b}$ closed.

\section{Conclusion}

Vague soft topology is an extension of fuzzy soft topology. Fuzzy soft topology is directly an extension of the crisp topology which has membership functional candidates bound by length one. However, it has a shortcoming, i.e., it only addresses a membership value and is unable to address the nonmembership value. Vague soft topology is dominant over fuzzy soft topology because it supposes all the two information that is true and false at the same time. Vague soft topology has narrowed domain as compared to vague soft bitopology. Some problems are very hard to discuss in vague soft topology. So, need of vague soft bitopology is felt. In our work, we regenerated some structures in vague soft bitopological spaces with new definition, that is, $*_{b}$ open sets concerning soft points. In future, we would try to see the validity of the given structures in vague soft tritopological structures under soft points of the space under the application of some more generalized vague soft open sets.

\section{Data Availability}

No data were used to support this study.

\section{Conflicts of Interest}

The authors declare that they have no conflicts of interest.

\section{Authors' Contributions}

All authors read and approved the final manuscript. 


\section{References}

[1] L. A. Zadeh, "Fuzzy sets," Information and Control, vol. 8, no. 3, pp. 338-353, 1965.

[2] K. T. Atanassov, "Intuitionistic fuzzy sets," Fuzzy Sets and Systems, vol. 20, no. 1, pp. 87-96, 1986.

[3] D. Molodtsov, "Soft set theory-first results," Computers \& Mathematics with Applications, vol. 37, no. 4/5, pp. 19-31, 1999.

[4] D. Molodtsov, The Theory of Soft Sets, URSS Publishers, Moscow, Russia, in Russian, 2004.

[5] W.-L. Gau and D. J. Buehrer, "Vague sets," IEEE Transactions on Systems, Man, and Cybernetics, vol. 23, no. 2, pp. 610-614, 1993.

[6] X. Wei, M. Jian, S. Wang, and G. Hao, "Vague soft sets and their properties," Computers \& Mathematics with Applications, vol. 59, pp. 787-794, 2010.

[7] X. Huang, H. Li, and Y. Yin, "Notes on "Vague soft sets and their properties"," Computers \& Mathematics with Applications, vol. 64, no. 6, pp. 2153-2157, 2012.

[8] C. Wang and Y. Li, "Topological structure of vague soft sets," Abstract and Applied Analysis, vol. 2014, Article ID 504021, 8 pages, 2014.

[9] A. Mukherjee and R. Das, "Neutrosophic bipolar vague soft set and its application to decision making problems," Neutrosophic Sets and Systems, vol. 32, p. 1, 2020.

[10] A. Al-Quran and N. Hassan, "Neutrosophic vague soft set and its applications," Malaysian Journal of Mathematical Sciences, vol. 11, no. 2, pp. 141-163, 2017. 